\title{
TGF- $\beta$-induced epigenetic deregulation of SOCS3 facilitates STAT3 signaling to promote fibrosis
}

\author{
Clara Dees, ${ }^{1}$ Sebastian Pötter, ${ }^{1}$ Yun Zhang, ${ }^{1}$ Christina Bergmann, ${ }^{1}$ Xiang Zhou, ${ }^{1}$ Markus Luber, ${ }^{1}$ Thomas Wohlfahrt, ${ }^{1}$ \\ Emmanuel Karouzakis, ${ }^{2}$ Andreas Ramming, ${ }^{1}$ Kolja Gelse, ${ }^{3}$ Akihiko Yoshimura, ${ }^{4}$ Rudolf Jaenisch,,${ }^{5}$ Oliver Distler, ${ }^{2}$ \\ Georg Schett, ${ }^{1}$ and Jörg H.W. Distler ${ }^{1}$ \\ 'Department of Internal Medicine 3 - Rheumatology and Immunology, Friedrich-Alexander-Universität (FAU) Erlangen-Nürnberg and Universitätsklinikum Erlangen, Erlangen, Germany. ${ }^{2}$ Center of \\ Experimental Rheumatology, Department of Rheumatology, University Hospital of Zurich, Zurich, Switzerland. 'Department of Trauma Surgery - Orthopedic Surgery, University of Erlangen-Nuremberg, \\ Erlangen, Germany. ${ }^{4}$ Department of Microbiology and Immunology, Keio University School of Medicine, Tokyo, Japan. ${ }^{5}$ Whitehead Institute for Biomedical Research, Cambridge, Massachusetts, USA
}

\begin{abstract}
Fibroblasts are key effector cells in tissue remodeling. They remain persistently activated in fibrotic diseases, resulting in progressive deposition of extracellular matrix. Although fibroblast activation may be initiated by external factors, prolonged activation can induce an "autonomous," self-maintaining profibrotic phenotype in fibroblasts. Accumulating evidence suggests that epigenetic alterations play a central role in establishing this persistently activated pathologic phenotype of fibroblasts. We demonstrated that in fibrotic skin of patients with systemic sclerosis (SSc), a prototypical idiopathic fibrotic disease, TGF- $\beta$ induced the expression of DNA methyltransferase 3A (DNMT3A) and DNMT1 in fibroblasts in a SMADdependent manner to silence the expression of suppressor of cytokine signaling 3 (SOCS3) by promoter hypermethylation. Downregulation of SOCS3 facilitated activation of STAT3 to promote fibroblast-to-myofibroblast transition, collagen release, and fibrosis in vitro and in vivo. Reestablishment of the epigenetic control of STAT3 signaling by genetic or pharmacological inactivation of DNMT3A reversed the activated phenotype of SSc fibroblasts in tissue culture, inhibited TCF- $\beta$-dependent fibroblast activation, and ameliorated experimental fibrosis in murine models. These findings identify a pathway of epigenetic imprinting of fibroblasts in fibrotic disease with translational implications for the development of targeted therapies in fibrotic diseases.
\end{abstract}

\section{Introduction}

Fibrotic tissue remodeling imposes a major burden on modern societies and has been estimated to contribute to up to $45 \%$ of deaths in the developed world and to cause socioeconomic costs on the order of tens of billions of dollars per year $(1,2)$. Fibrotic tissue remodeling may occur in response to trauma, infection/ inflammation, or tumors, but in many cases, no initiating triggers can be identified. Systemic sclerosis (SSc) is a prototypical example of such an idiopathic fibrotic disease (3). Failure of the affected organs is common in SSc and results in high morbidity and mortality, particularly because no targeted therapies are yet available for the treatment of fibrosis $(3,4)$. Aberrant activation of resident fibroblasts and their transition into myofibroblasts is the common denominator of fibrotic diseases (2-4). Overwhelming evidence highlights TGF- $\beta$ as a core mediator of fibroblast activation (3-7). TGF- $\beta$ signaling is chronically activated in SSc and other fibrotic diseases, and activated fibroblasts demonstrate a TGF- $\beta$-biased gene expression signature that persists in vitro (8-11). Moreover, TGF- $\beta$ is sufficient to induce myofibroblast differentiation in vitro and tissue fibrosis in vivo (12).

Conflict of interest: The authors have declared that no conflict of interest exists. Copyright: (5) 2020, American Society for Clinical Investigation.

Submitted: May 25, 2018; Accepted: January 17, 2020; Published: April 6, 2020.

Reference information: J Clin Invest. 2020;130(5):2347-2363.

https://doi.org/10.1172/JCl122462.
Although myofibroblasts are only transiently observed during physiological wound healing, they persist in fibrotic diseases, resulting in persistent repair responses and progressive tissue fibrosis. More than 40 years ago, LeRoy and colleagues provided first evidence for an endogenous activation of SSc fibroblasts, demonstrating that SSc fibroblasts maintain an activated phenotype in vitro, even in the absence of other cell types or constituents of the profibrotic milieu in SSc (13). Endogenous activation of TGF- $\beta$ in SSc fibroblasts in cell culture is critical to maintaining this persistently activated phenotype (14). This finding suggested that, although myofibroblast differentiation may initially be driven by external factors, prolonged activation could render them autonomous and independent of external stimuli.

Changes in the epigenetic code are central for establishing and maintaining the activated phenotype of fibroblasts in fibrotic diseases (15-20). Among the different epigenetic modifications, DNA methylation remains most intensely studied. Methylation of DNA occurs on position 5 of the pyrimidine ring of cytosine residues within $\mathrm{CpG}$ dinucleotides and leads to transcriptional silencing (21). A family of DNA methyltransferases (DNMT) comprising 3 members in mammals controls DNA methylation: DNMT1, which is predominantly a maintenance methyltransferase with a preference for hemimethylated sites, and DNMT3A and DNMT3B, which are thought to function mainly as de novo DNMTs (21-23). DNA methylation may drive fibrosis by silencing of antifibrotic genes (24-28), as evidenced by downregulation of 

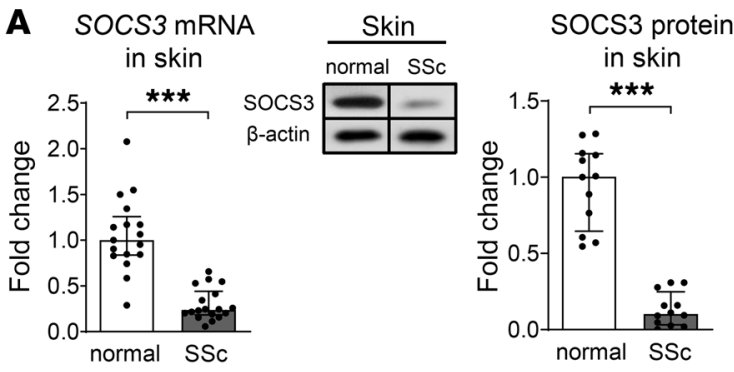

B
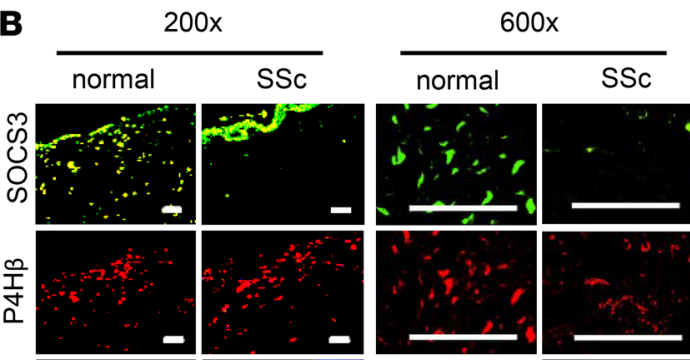

SOCS3-positive

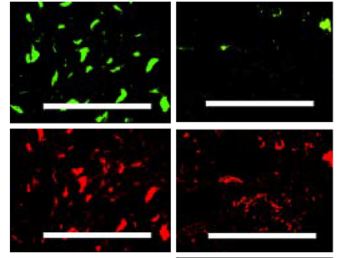
fibroblasts
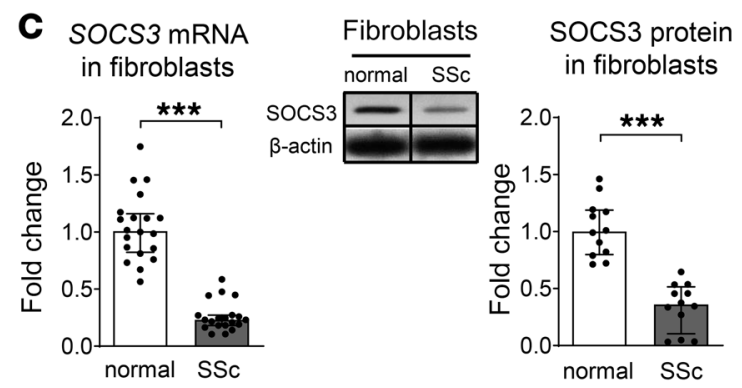

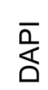

normal SSc in fibroblasts
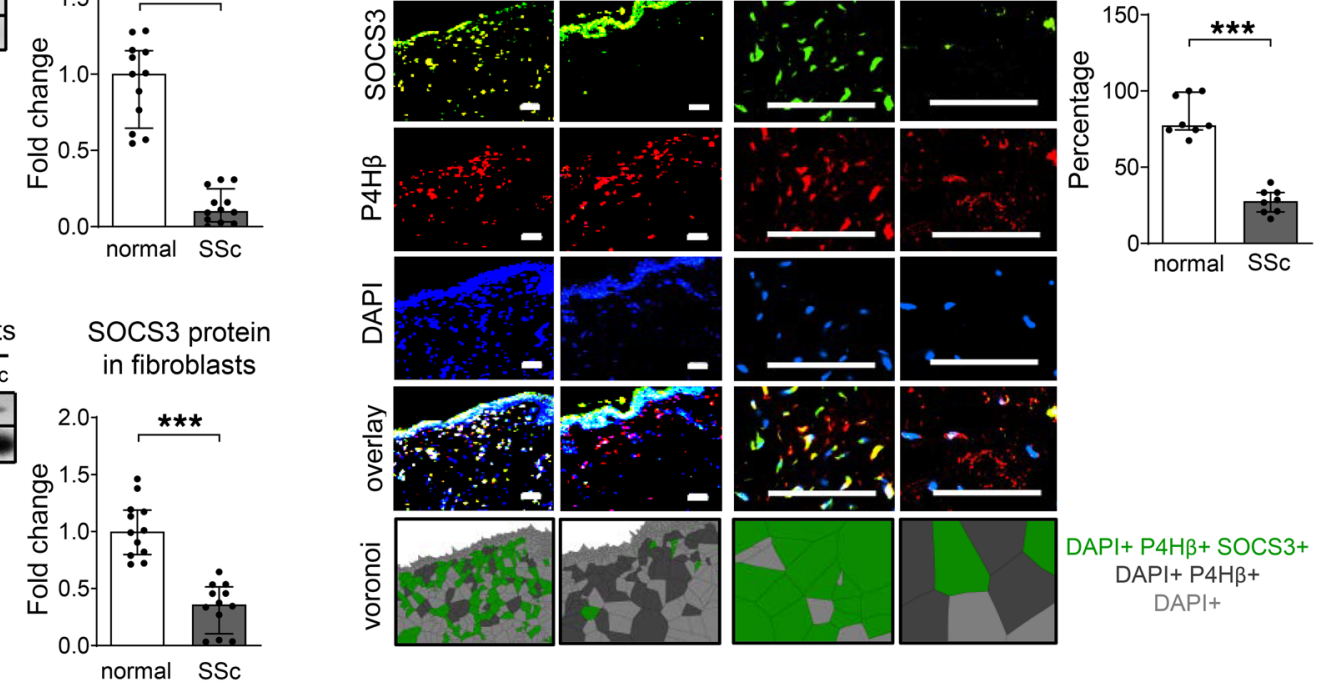

D
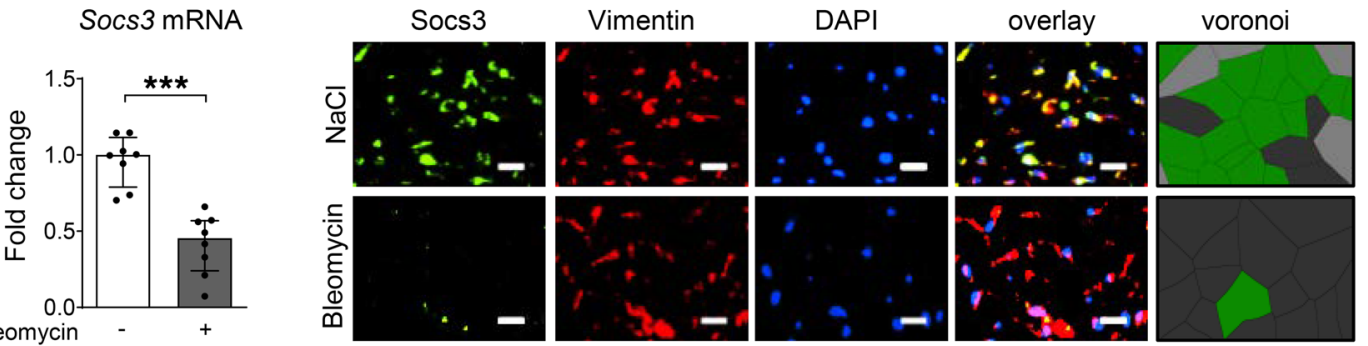

DAPI+ vimentin + SOCS3+ $\mathrm{DAPI}+\mathrm{P} 4 \mathrm{H} \beta+$ DAPI+

E

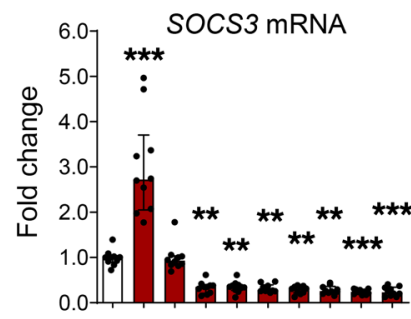

hours - 14461216244872120 TGF $\beta$
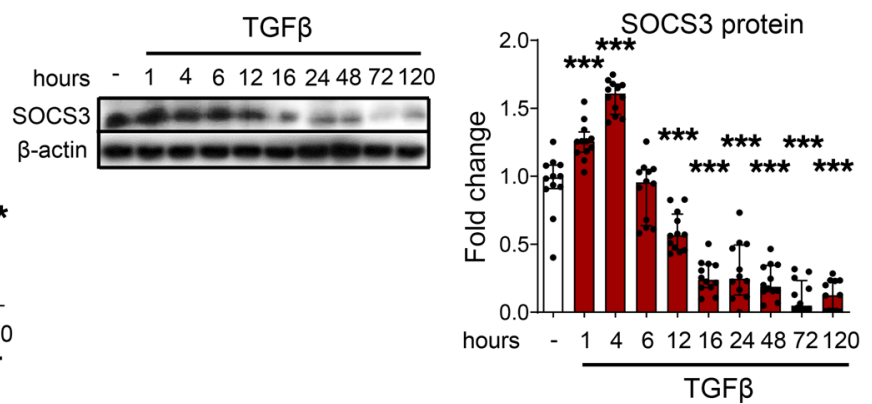

$\mathbf{F}$
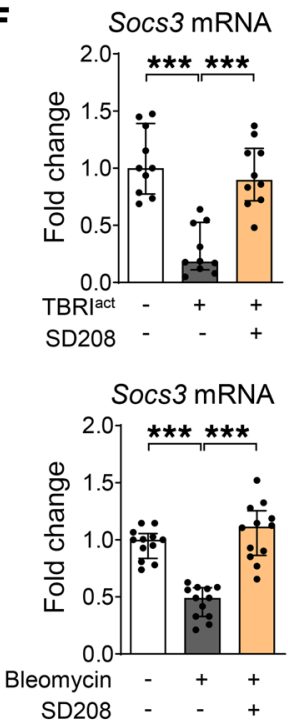
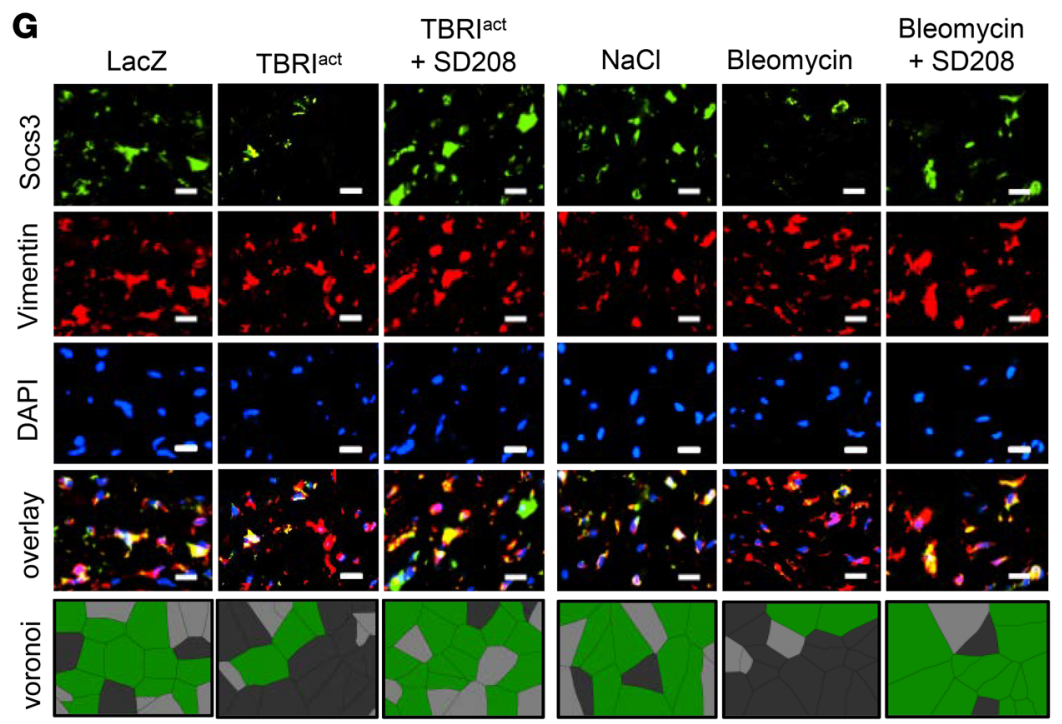

DAPI+ P4HB + SOCS3+ $\mathrm{DAPI}+\mathrm{P} 4 \mathrm{H} \beta+$ $\mathrm{DAPI}+$ 
Figure 1. Decreased expression of SOCS3 in SSc and in experimental fibrosis. (A) Expression of SOCS3 in SSc and normal human skin. $n=9$ skin samples/group for qPCR with 2 technical replicates each; $n=4$ skin samples/group for Western blot with 3 technical replicates each. (B) Representative fluorescence costaining for SOCS3 (green) with $\mathrm{P} 4 \mathrm{H} \beta$ (red) and DAPI (blue) in skin sections of SSc patients and healthy controls and voronoi tessellation visualizing single-, double- and triple-positive cells. $n=8$ skin samples/group. Scale bars: $250 \mu \mathrm{m}$. (C) Expression of SOCS3 in cultured human fibroblasts. $n=10$ fibroblast lines from different donors for qPCR with 2 technical replicates each; $n=4$ fibroblast lines from different donors for Western blot with 3 technical replicates each. (D) Socs3 expression in bleomycin-induced dermal fibrosis: mRNA levels and representative images of immunofluorescence staining together with voronoi tesselation visualizing single-, double-, and triple-positive cells for Socs3 and vimentin. Original magnification, $\times 600 . n=8$ mice per group. Scale bars: $50 \mu \mathrm{m}$. (E) SOCS3 expression in normal human fibroblasts upon chronic stimulation with TCF- $\beta . n=5$ fibroblast lines from different donors for qPCR with 2 technical replicates each; $n=4$ fibroblast lines from different donors for Western blot with 3 technical replicates each. (F) mRNA levels of Socs3 in TBRIact - and bleomycin-induced fibrosis treated with the TBRI inhibitor SD-208. $n=5$ mice/group with 2 technical replicates each. (C) Representative immunofluorescence stainings with voronoi tessellation visualizing single-, double-, and triple-positive cells for Socs 3 and vimentin in the murine models of bleomycin- and TBRI act-induced dermal fibrosis with cotreatment with SD-208. $n=5$ mice/group. Original magnification, $\times 600$. Scale bars: $50 \mu \mathrm{m}$. Data are depicted as the median with interquartile range. Each dot represents an individual result. Mann-Whitney $U$ test (A-D) or 1-way ANOVA with Tukey's range test as post hoc analysis (E and $\mathbf{F}$ ) was used for statistical analyses. $0.01>{ }^{* *} P \geq 0.001 ;{ }^{* *} P<0.001$.

the antifibrotic transcription factors FLI1 and $\operatorname{KLF} 5$ in SSc $(29,30)$. Those findings are of therapeutic interest, as epigenetic changes are reversible. DNMTs can be targeted by small molecule inhibitors, such as 5-azacitidine and 5-aza-2'-deoxycytidine (5-aza), which are already approved for clinical use in myelodysplastic syndrome and acute myeloid leukemia.

JAK/STAT signaling pathways are well established as crucial mediators of inflammation $(31,32)$. Small molecule inhibitors of JAKs are approved for clinical use in rheumatoid arthritis and await approval for other inflammatory diseases. In fibrotic diseases, however, the role of JAK/STAT signaling is not restricted to the regulation of inflammation. We and others have demonstrated recently that JAK2/STAT3 signaling also transmits the profibrotic effects of TGF- $\beta$ on fibroblasts and that targeted inhibition of JAK2 or STAT3 ameliorates fibroblast activation and fibrosis (33-35). JAK2/STAT3 may thus not only modulate fibrotic tissue remodeling by regulating inflammatory responses, but also by directly controlling fibroblast activation. Two members of the suppressor of cytokine signaling (SOCS) proteins, SOCS1 and SOCS3, are crucial regulators of JAK/STAT signaling. The family of SOCS proteins consists of 8 members, SOCS1 to SOCS7 and cytokine-induced $\mathrm{SH} 2$ containing protein (CIS), which share a central SH2 domain and a SOCS box at the C-terminus. However, only SOCS1 and SOCS3 possess an N-terminal kinase inhibitory region (KIR) domain, which enables them to block the transfer of phosphate from ATP to STAT proteins (36-38). SOCS1 and SOCS3 can thus act as noncompetitive inhibitors of JAKs. In addition, SOCS proteins may also induce degradation of JAKs and of the associated cytokine receptors (39). Of note, SOCS proteins are transcriptional targets of STAT proteins: activation of STAT signaling induces the expression of SOCS proteins to provide a negative feedback loop (39). This endogenous feedback regulation is critical to preventing aberrant activation of JAK/STAT signaling. Loss-of-function mutations of SOCS proteins with consecutive activation of JAK/ STAT signaling have been observed in different hematologic and solid tumors (40-43). However, more commonly than by somatic mutations, tumor cells escape the endogenous regulation of JAK/STAT signaling by epigenetic imprinting of SOCS genes (44). Hence, in various types of B cell neoplasias and carcinomas, the expression of SOCS1 or SOCS3 is silenced by DNA hypermethylation $\left(43,45^{-58}\right)$.

In the present study, we tested the hypothesis that epigenetic silencing of SOCS expression may contribute to the aberrant activation of JAK2/STAT3 signaling by TGF- $\beta$ in SSc and that reestablishment of the endogenous, SOCS-dependent control of JAK/ STAT signaling may prevent aberrant fibroblast activation and ameliorate tissue fibrosis.

\section{Results}

The expression of SOCS3 is downregulated in SSc and in experimental skin fibrosis in a TGF- $\beta$-dependent manner. We first analyzed the expression of SOCS proteins in fibrotic skin of SSc patients. The mRNA and protein levels of SOCS3 were decreased in SSc skin as compared with that of healthy individuals of the same age and sex (Figure 1A). We also found reduced staining for SOCS3 in fibroblasts in SSc skin by costaining for SOCS3 and the fibroblast marker prolyl-4-hydroxylase- $\beta$ (P4H $\beta$, also known as PDI) (Figure 1B). The downregulation of SOCS3 persisted in cultured SSc fibroblasts with decreased mRNA and protein levels of SOCS3 as compared with fibroblasts from normal skin (Figure $1 C)$. The reduction of SOCS3 protein levels and of the number of $\mathrm{SOCS3}^{+}$fibroblasts was more pronounced in diffuse-cutaneous SSc than in limited cutaneous SSc (Supplemental Figure 1; supplemental material available online with this article; https:// doi.org/10.1172/JCI122462DS1). The expression of SOCS3 was also reduced in experimental fibrosis with decreased staining in vimentin-positive cells in the skin of mice challenged with bleomycin as compared with nonfibrotic controls (Figure 1D). In contrast to SOCS3, we did not observe changes in SOCS1 expression in SSc or in experimental dermal fibrosis (data not shown), indicating a SOCS3-specific deregulation.

Given the consistent downregulation of SOCS3 expression in human SSc skin, in cultured SSc fibroblasts, and in experimental fibrosis, we hypothesized that a core pathway of fibrosis, such as TGF- $\beta$, may regulate SOCS3 expression. Stimulation of cultured fibroblasts with recombinant TGF- $\beta$ induced an early, transient upregulation of SOCS3 (Figure 1E). However, with prolonged follow-up, the mRNA and protein levels of SOCS3 declined strongly to below baseline levels. The downregulation of SOCS3 was maintained and SOCS3 expression remained stably suppressed when fibroblasts were exposed to persistently increased levels of TGF- $\beta$ as in fibrotic tissues (Figure 1E). In contrast, incubation of SSc fibroblasts with SD-208, a selective inhibitor of TGF- $\beta$ receptor I kinase activity, increased SOCS3 expression to levels that were comparable to those of normal fibroblasts (Supplemental Figure 2A). The regulatory effects of TGF- $\beta$ on SOCS3 were confirmed in vivo. Activation of TGF- $\beta$ signaling by overexpression of a constitutively 
A

SOCS3 mRNA

(SSc fibroblasts)

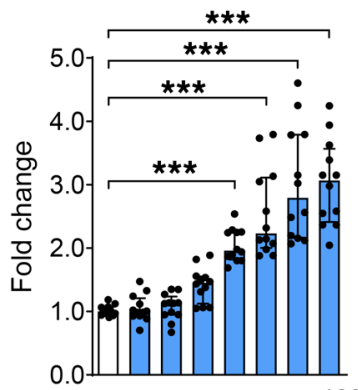

hours - 2612247296120

5-aza

B $\quad$ socs3 mRNA

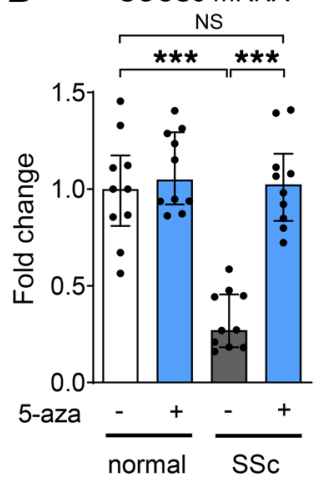

SOCS3 protein

(SSc fibroblasts)

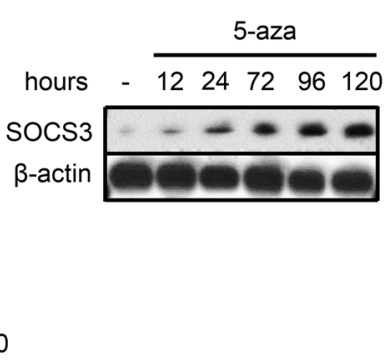

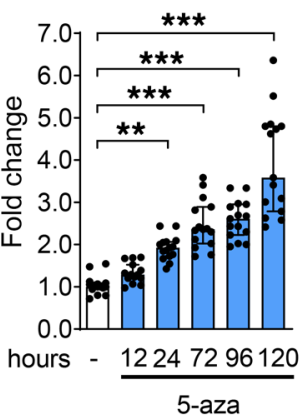

SOCS3 protein

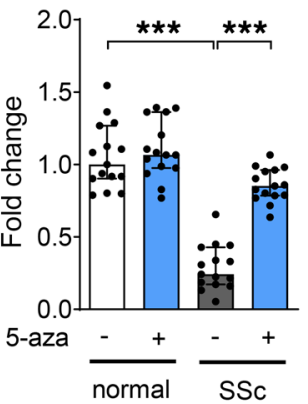

C

SOCS3 methylation in meth.-spec. PCR

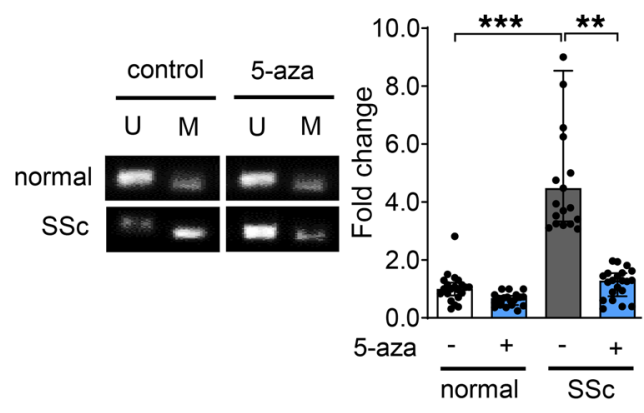

SOCS3 methylation in MeDIP

Fold enrichment

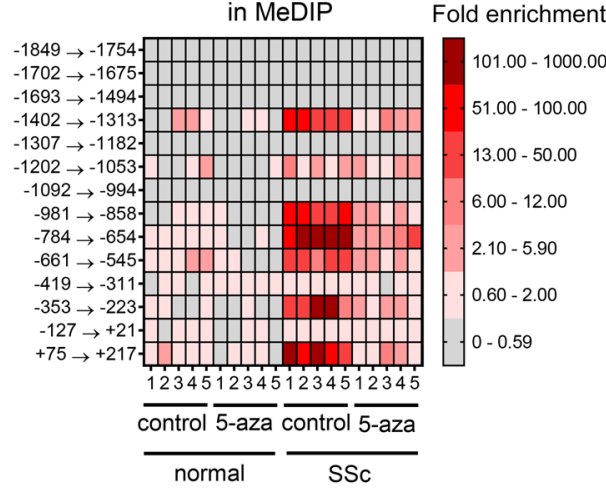

$\mathbf{E}$

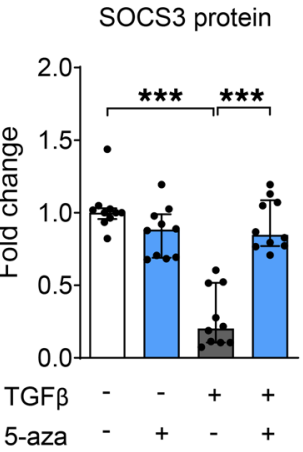

Socs 3 mRNA
Socs 3 protein

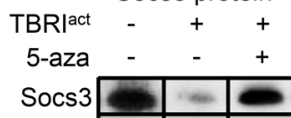

$\beta$-actin $\square$

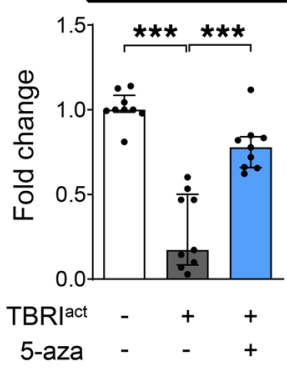

5-aza

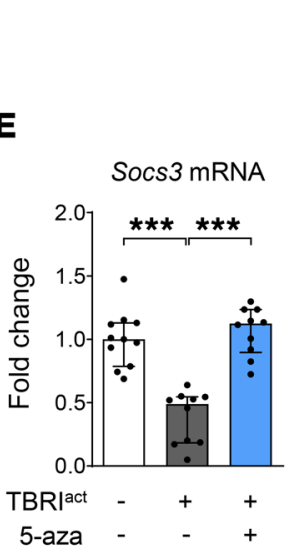

$\mathbf{F}$

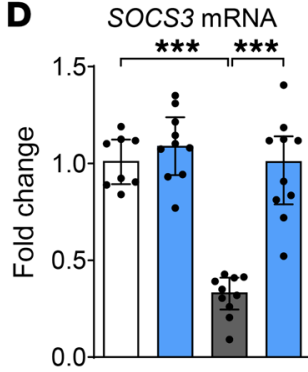

TGF $\beta$

5-aza

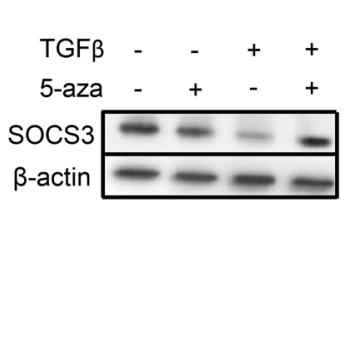

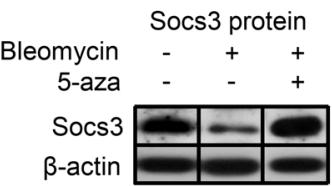

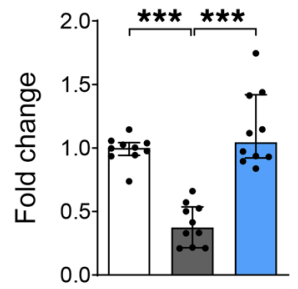

Bleomycin

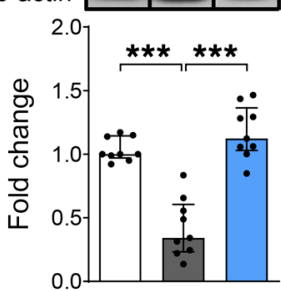

Bleomycin - + +

5-aza - - +
G

SOCS3 methylation in meth.-spec. PCR
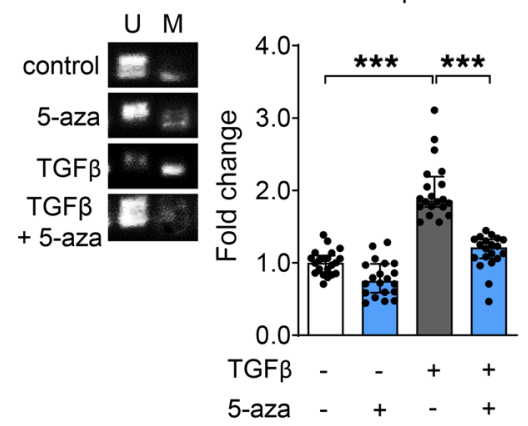

SOCS3 methylation in MeDIP

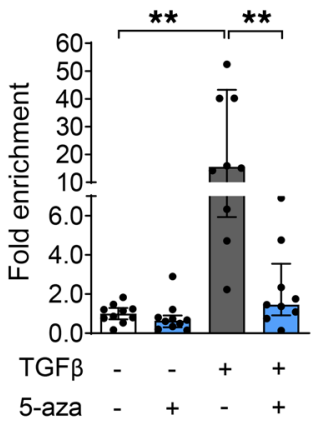


Figure 2. Regulation of SOCS3 expression by DNA methylation. (A) SOCS3 expression in SSc fibroblasts incubated with 5 -aza. $n=6$ fibroblast lines from different donors for QPCR with 2 technical replicates each; $n=5$ fibroblast lines from different donors for Western blot with 3 technical replicates each. (B) Effects of 5-aza on SOCS3 expression in SSc and normal fibroblasts. $n=5$ fibroblast lines from different donors for qPCR with 2 technical replicates each; $n=5$ fibroblast lines from different donors for Western blot with 3 technical replicates each. (C) Promoter methylation of SOCS3 by methylation-specific PCR (MSP) (U, unmethylated; M, methylated PCR) and MeDIP assay in SSc and normal fibroblasts incubated with 5 -aza. $n=7$ fibroblast lines from different donors for MSP with 3 technical replicates each; $n=5$ fibroblast lines from different donors for MeDIP with 4 technical replicates each. (D) SOCS3 expression in normal fibroblasts stimulated with TGF- $\beta$ and incubated with 5 -aza. $n=5$ fibroblast lines from different donors for both qPCR and Western blot with 2 technical replicates each. (E and $\mathbf{F}$ ) Socs3 expression in (E) TBR lact- and (F) bleomycin-induced fibrosis treated with 5-aza. $n=5$ mice/group for qPCR with 2 technical replicates each; $n=3$ mice/group for Western blot with 3 technical replicates each. (G) Promoter methylation of SOCS3 induced by TCF- $\beta$ in normal human fibroblasts as analyzed by MSP and MeDIP assay. $n=10$ fibroblast lines from different donors for MSP with 2 technical replicates each; $n=5$ fibroblast lines from different donors for MeDIP with 4 technical replicates each. Data are depicted as the median with interquartile range. Each dot represents an individual result. One-way ANOVA with Tukey's range test as post hoc analysis was used for statistical analyses. $0.01>{ }^{* *} P \geq 0.001 ;{ }^{* *} P<0.001$

active TGF- $\beta$ receptor type I (TBRI ${ }^{\text {act})}$ decreased the expression of Socs 3 in murine skin (Figure 1, F and G). Moreover, the downregulation of Socs 3 in bleomycin- and TBRI ${ }^{\text {act-induced fibrosis was }}$ blocked by selective inhibition of TGF- $\beta$ signaling with SD-208 (Figure 1, F and G), confirming that TGF- $\beta$ is both sufficient and required to downregulate SOCS3 expression in skin fibrosis.

TGF- $\beta$ inhibits SOCS3 expression by hypermethylation of the SOCS3 promoter. The findings of a persistent downregulation of SOCS3 expression in cultured SSc fibroblasts and the repression of SOCS3 upon stimulation with TGF- $\beta$ suggested that epigenetic mechanisms might account for the reduced expression of SOCS3. In cancer cells, the transcription of SOCS3 has been shown to be regulated by DNA methylation $(54,59)$. To evaluate whether DNA methylation is implicated in the downregulation of SOCS3 expression in SSc, we first inhibited the activity of DNMT by 5-aza. In SSc fibroblasts, incubation with 5-aza increased the mRNA and protein levels of SOCS3 in a time-dependent manner (Figure 2A). In contrast, 5-aza had no significant effects on the steady-state expression of SOCS3 in fibroblasts derived from healthy individuals ("normal" fibroblasts) (Figure 2B). To directly demonstrate hypermethylation of the promoter region of the SOCS3 gene in SSc fibroblasts, we performed methylation-specific PCR. The ratio of methylated to unmethylated DNA was higher in fibroblasts from SSc skin compared with fibroblasts isolated from the skin of healthy individuals (Figure 2C). Methylated DNA immunoprecipitation (MeDIP) analysis of 14 sites throughout the $\mathrm{CpG}$ island in the promoter of the SOCS3 gene demonstrated that hypermethylation occurs in particular at -1402 to $-1313,-981$ to $-545,-784$ to $-654,-661$ to $-545,-353$ to -223 , and +75 to +217 with $40-$ to 100 -fold increases in SSc fibroblasts as compared with fibroblasts from healthy individuals. These sites were consistently hypermethylated in all lines of fibroblasts derived from patients with diffuse-cutaneous SSc (Figure 2C and Supplemental Figure 3).
We also detected low levels of methylation at sites -1202 to -1053 , -419 to -311 , and -127 to +21 , with trends toward slight increases in SSc fibroblasts (Figure 2C and Supplemental Figure 3). Consistent with the lack of differences in expression of SOCS1, we did not observe differences in the methylation status of the promoter of SOCS1 in SSc fibroblasts (data not shown).

We next investigated whether epigenetic imprinting by promoter hypermethylation also accounts for the repression of SOCS3 expression upon prolonged stimulation with TGF- $\beta$. Indeed, incubation with 5-aza prevented the downregulation of SOCS3 mRNA and protein in dermal fibroblasts from healthy donors upon prolonged exposure to TGF- $\beta$ (Figure 2D). However, 5-aza did not further increase the levels of SOCS3 in SSc fibroblasts preincubated for extended periods with SD-208 (Supplemental Figure $2 \mathrm{~A}$ ), highlighting that the endogenous downregulation of SOCS3 in SSc is dependent on the well-known hyperactive TGF- $\beta$ signaling in these cells (14). Moreover, repression of SOCS3 in the skin of TBRI ${ }^{\text {act }}$ mice was reversed by the cotreatment with 5-aza (Figure $2 \mathrm{E}$ ). Similar results were obtained in bleomycin-induced skin fibrosis (Figure 2F). Consistent with the findings obtained with 5-aza, methylation-specific PCR and MeDIP assays demonstrated an upregulation of DNA methylation at the SOCS3 promoter at -784 to -654 , the most highly methylated site in SSc fibroblasts, by TGF- $\beta$ in normal fibroblasts, which was prevented by coincubation with 5-aza (Figure $2 \mathrm{G}$ ).

To determine whether TGF- $\beta$ promotes DNA methylation by regulation of DNMT expression, we monitored the expression levels of all 3 DNMTs in fibroblasts stimulated with TGF- $\beta$ over time. TGF- $\beta$ upregulated the expression of DNMT1 and DNMT3A, but had no effect on the mRNA and protein levels of DNMT3B (Figure 3A). Notably, the kinetics of the induction of DNMT1 and DNMT3A differed. First effects of TGF- $\beta$ on DNMT3A expression occurred within 6 hours, with significant effects after 12 hours, while an induction of DNMT1 did not occur before 72 hours (Figure 3A). The differences in the kinetics suggest an indirect induction of DNMT1, whereas DNMT3A may be regulated directly by TGF- $\beta$. Indeed, the upregulation of DNMT3A mRNA by TGF- $\beta$ did not require de novo protein synthesis, as analyzed by coincubation with cycloheximide (data not shown). Stimulation with TGF- $\beta$ also induced DNMT activity in fibroblasts, with first effects within 4 hours (Figure $3 \mathrm{~B}$ ).

siRNA-mediated knockdown of SMAD3 and SMAD4 demonstrated that TGF- $\beta$ induces DNMT3A by canonical TGF- $\beta$ signaling (Supplemental Figure 2, B and C). In contrast, targeted inhibition of noncanonical TGF- $\beta$ downstream mediators such as JNK, p38, Ras, or Rac had no effect (data not shown). To further confirm the direct regulation of DNMT3A expression by TGF- $\beta$-induced SMAD3 signaling, we analyzed the DNMT3A promoter for potential SMAD3-binding sites and performed ChIP assay. In silico analyses of the DNMT3A promoter revealed 4 SMAD-binding elements (SBE). ChIP assays demonstrated that TGF- $\beta$ induced SMAD3 binding to the DNMT3A promoter at -3423 bp (Supplemental Figure 2D). Thus, TGF- $\beta$ regulates DNA methylation in fibroblasts by SMAD3-dependent upregulation of DNMT3A expression.

The different kinetics of DNMT1 and DNMT3A induction by TGF- $\beta$ may also reflect the functional differences of both DNMTs, with DNMT3 acting predominantly as a de novo DNMT and DNMT1 rather maintaining established DNA methylation (21). 
A DNMT1 mRNA

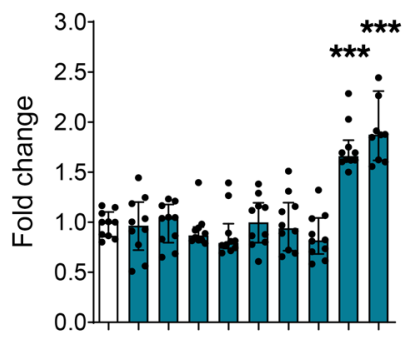

hours $-1 \quad 4 \quad 6 \quad 1216244872120$ TGF $\beta$

DNMT3A mRNA
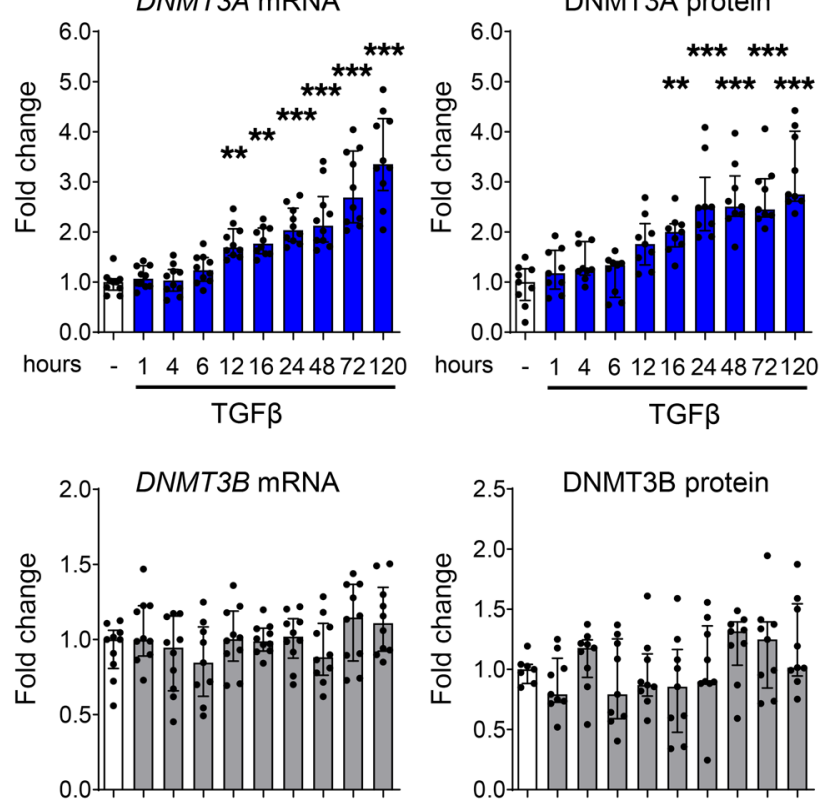

hours

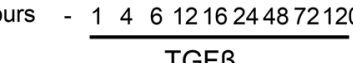

0

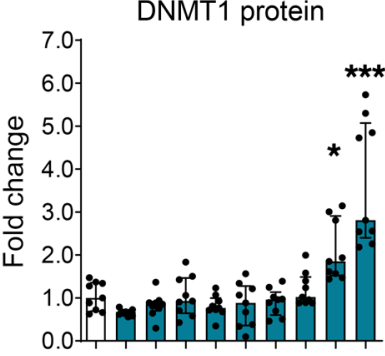

hours

1461216244872120

TGF $\beta$

DNMT3A protein

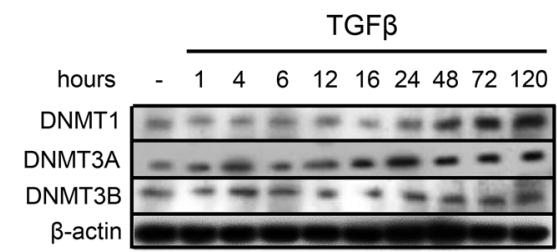

E

DNMT mRNA in skin 口normal $\square S S c$

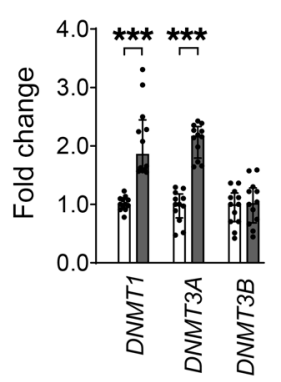

DNMT protein in skin

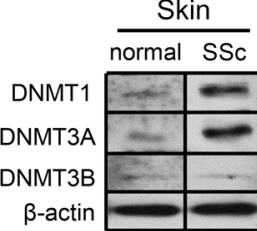

$\square$ normal $\square \mathrm{SSc}$
TGF $\beta$

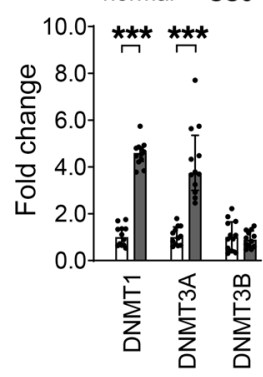

B DNMT activity

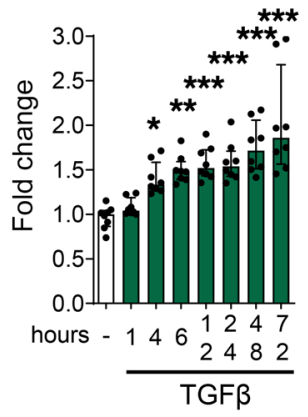

D $\quad$ SOCS3 mRNA (SSc fibroblasts)

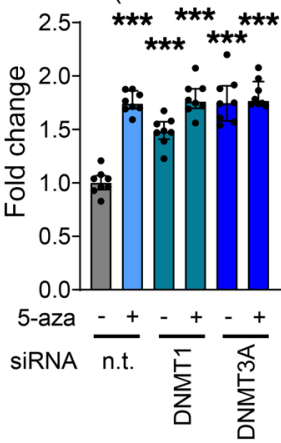

C SOCS3 mRNA

DNMT3A MRNA
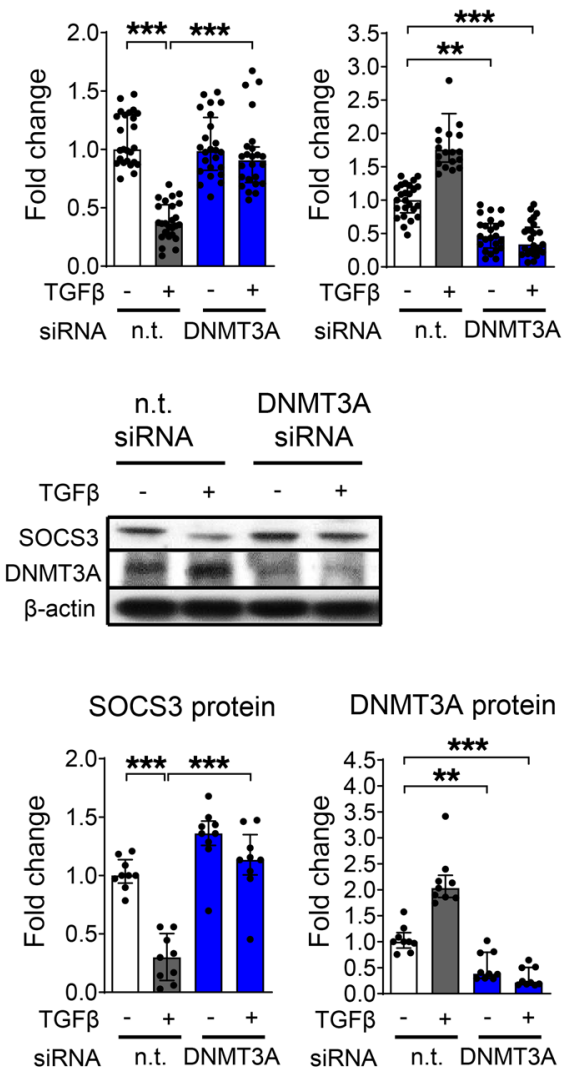

F $\quad D N M T$ mRNA

DNMT protein in fibroblasts

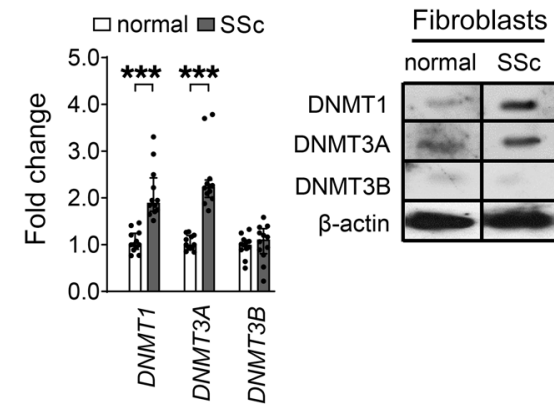

口normal $\square$ SSC

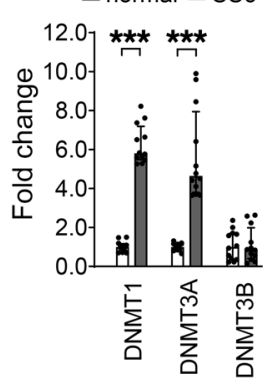


Figure 3. Dysregulated expression of DNMT3A. (A) mRNA and protein levels of DNMT1, DNMT3A, and DNMT3B upon chronic stimulation with TCF- $\beta$ in normal dermal fibroblasts. $n=5$ fibroblast lines from different donors with 2 technical replicates each for qPCR; $n=3$ fibroblast lines from different donors with 3 technical replicates each for Western blot. (B) Activity of DNMTs upon chronic stimulation with TCF- $\beta . n=4$ fibroblast lines from different donors with 2 technical replicates each. (C) mRNA and protein levels of SOCS3 and DNMT3A upon knockdown of DNMT3A in TGF- $\beta$-stimulated normal fibroblasts. $n=12$ fibroblast lines from different donors for qPCR with 2 technical replicates each; $n=3$ fibroblast lines from different donors for Western blot with 3 technical replicates each. (D) Expression of SOCS3 upon knockdown of DNMT1 or DNMT3A in SSc fibroblasts. $n=4$ fibroblast lines from different donors with 2 technical replicates each. (E and $\mathbf{F})$ mRNA and protein levels of DNMT1, DNMT3A, and DNMT3B in (E) human skin and $(\mathbf{F})$ human cultured fibroblasts. $n=6$ fibroblast lines or skin samples from different donors for QPCR with 2 technical replicates each; $n=4$ fibroblast lines or skin samples from different donors for Western blot with 3 technical replicates each. Data are depicted as the median with interquartile range. Each dot represents an individual result. One-way ANOVA with Tukey's range test as post hoc analysis (A-D) or Mann-Whitney $U$ test ( $E$ and $\mathbf{F}$ ) was used for statistical analyses. $0.05>{ }^{*} P \geq 0.01 ; 0.01$ ${ }^{* *} P \geq 0.001 ;{ }^{* *} P<0.001$.

Consistent with this interpretation, individual knockdown of DNMT3A, but also of DNMT1, was each sufficient to completely prevent the downregulation of SOCS3 upon prolonged incubation with TGF- $\beta$ in normal fibroblasts and to reactivate the baseline expression of SOCS3 in SSc fibroblasts (Figure 3, C and D).

In line with increased TGF- $\beta$ signaling in fibrotic tissues and the endogenous activation of TGF- $\beta$ signaling in cultured SSc fibroblasts, we observed increased mRNA and protein levels of DNMT1 and DNMT3A, but not of DNMT3B, in SSc skin and in SSc fibroblasts compared with matched healthy skin and normal dermal fibroblasts, respectively (Figure 3, E and F). Moreover, incubation with SD-208 decreased DNMT1 and DNMT3A expression in SSc fibroblasts to levels comparable to those of normal fibroblasts, suggesting that the increased expression of DNMT1 and DNMT3A depends on autocrine TGF- $\beta$ signaling in SSc fibroblasts (Supplemental Figure 2E). Together, these data demonstrate that TGF- $\beta$ induces DNMT3A and DNMT1 in a SMAD-dependent manner to silence the expression of SOCS3 by promoter hypermethylation in SSc.

SOCS3 limits TGF- $\beta$-dependent fibroblast activation. To investigate the functional role of SOCS3 in fibroblast activation in SSc, we first targeted the expression of SOCS3 in fibroblasts. siRNAmediated knockdown of SOCS3 in dermal fibroblasts from healthy volunteers increased mRNA levels of COL1A1 and COL1A2, stimulated the release of collagen protein, and promoted the expression of $\alpha$-smooth muscle actin ( $\alpha$-SMA) and the formation of stress fibers to levels almost comparable to those of SSc fibroblasts (Figure $4 \mathrm{~A}$ and Supplemental Figure 4B). In contrast to the effects on normal fibroblasts, knockdown of SOCS3 had no further effects on collagen release or the expression of myofibroblast markers in SSc fibroblasts, a finding that is in line with the promoter methylation-induced silencing of SOCS3 in SSc fibroblasts (Figure 4C and Supplemental Figure 4D).

Coincubation with 5-aza to prevent the downregulation of SOCS3 reduced the stimulatory effects of TGF- $\beta$ on normal fibroblasts and reduced the upregulation of COL1A1 and COL1A2 mRNA, collagen protein, $\alpha$-SMA, and fibers (Figure 4A and Sup- plemental Figure 4B), but had no significant effects on the basal expression levels in normal fibroblasts. Moreover, incubation with 5 -aza also reversed the activated phenotype of established myofibroblasts when applied 72 hours after first exposure to TGF- $\beta$ (Supplemental Figure 5). Incubation with 5-aza also inhibited the aberrant activation of SSc fibroblasts and reduced the release of collagen as well as the expression of myofibroblast markers (Figure $4 \mathrm{C}$ and Supplemental Figure 4D). Selective inactivation of DNMT3A or DNMT1 by siRNA-mediated knockdown also inhibited the TGF- $\beta$-induced activation of fibroblasts isolated from healthy donors and decreased the basal activation of SSc fibroblasts (Figure 4, B and D, and Supplemental Figure 4, C and D).

To exclude that the increased sensitivity of SSc fibroblasts to the antifibrotic effects of 5-aza is based on enhanced proliferation of these cells, all experiments were performed with confluent and thus contact-inhibited, nonproliferating cells. Moreover, coincubation with cell cycle inhibitors did not ameliorate the differences in responsiveness to 5 -aza between SSc fibroblasts and normal fibroblasts (data not shown).

In contrast, forced overexpression of SOCS3 inhibited TGF- $\beta$ induced fibroblast activation in normal fibroblasts with reduced induction of COL1A1 and COL1A2 mRNA as well as the release of collagen (Figure 5, A and B). Overexpression of SOCS3 also ameliorated the activated phenotype of SSc fibroblasts (Figure 5, C and D).

Inhibition of epigenetic silencing of Socs 3 ameliorates experimental fibrosis. To investigate the role of Socs3 in the pathogenesis of experimental fibrosis, we evaluated the outcome of mice with fibroblast-specific deletion of Socs3 (Socs $3^{\text {R/fl }} \mathrm{Col}$ CCre) in bleomycin- and

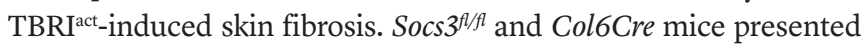
with normal skin architecture and responses to profibrotic stimuli comparable to those of WT mice (data not shown). Socs $3^{\text {f/l }}$ Col6Cre double-transgenic mice also showed a normal skin architecture under homeostatic conditions. However, Socs $3^{\text {f/l }}$ Col6Cre mice demonstrated enhanced responses to profibrotic stimuli (Figure 6, $\mathrm{A}-\mathrm{D}$, and Supplemental Figure 6A). In the mouse model of bleomycin-induced skin fibrosis, dermal thickening, myofibroblast accumulation, and hydroxyproline content were more pronounced in Socs $3^{f / f l}$ Col6Cre mice compared with Socs $3^{3 / f l}$ control mice (Figure 6 , A and B). Consistent with the regulatory effects of SOCS3 on TGF- $\beta$-induced fibroblast activation, Socs $3^{\nexists / / l}$ Col6Cre mice were

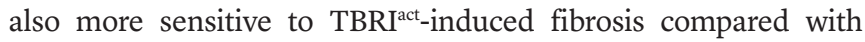
Socs $3^{3 / f l}$ mice (Figure 6, C and D).

To further highlight the role of DNA methylation-induced silencing of SOCS3 in the pathogenesis of skin fibrosis, we treated Socs $3^{n / f l}$ Col6Cre mice and control littermates with 5-aza. Treatment with 5-aza ameliorated bleomycin- and TBRI ${ }^{\text {act-induced skin }}$ fibrosis in Socs $3^{f / / l}$ control mice, with reduced dermal thickening, decreased myofibroblast counts, and lower hydroxyproline content (Figure 6, A-D). In control mice, treatment with 5-aza not only prevented fibrosis in control littermates, but also induced regression of preestablished bleomycin-induced fibrosis (Supplemental Figure 7). In contrast with what occurred in control mice, treatment with 5 -aza did not demonstrate pronounced antifibrotic effects in Socs $3^{3 / / /}$ Col6Cre mice (Figure 6, A-D), providing further evidence for the pathophysiologic relevance of the epigenetic deregulation of SOCS3 in fibrotic conditions. Treatment with 5-aza was well tolerated without evidence of weight loss or other signs of toxicity, such as chang- 
A

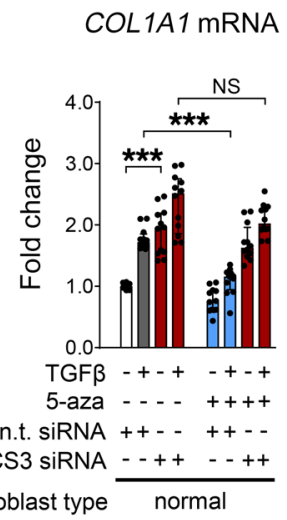

B

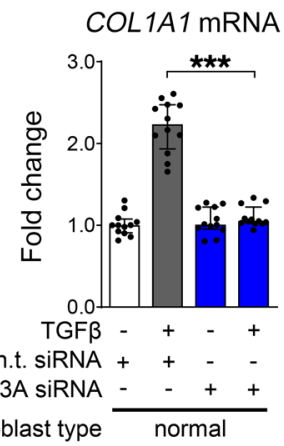

C

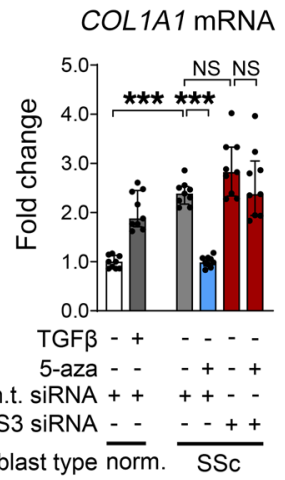

D
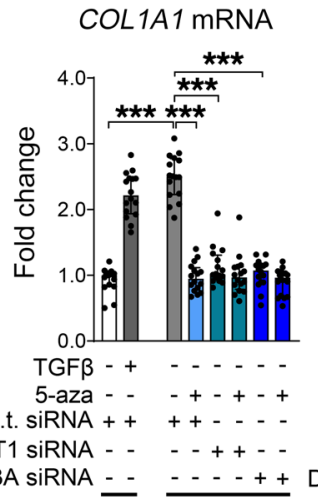

Fibroblast type norm. $\frac{-\cdots+++}{\text { SSc }}$
COL1A2 mRNA

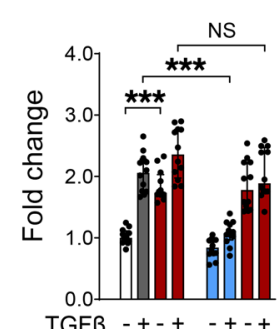

5-aza - - - ++++

n.t. SiRNA ++- + ++-

SOCS3 siRNA $-+++\quad-++$

Fibroblast type normal

COL1A2 mRNA

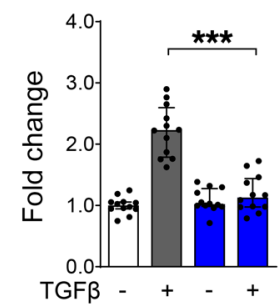

n.t. siRNA + + - -

DNMT3A siRNA $\frac{-++}{\text { normal }}$
TGF $\beta-+-+-++$
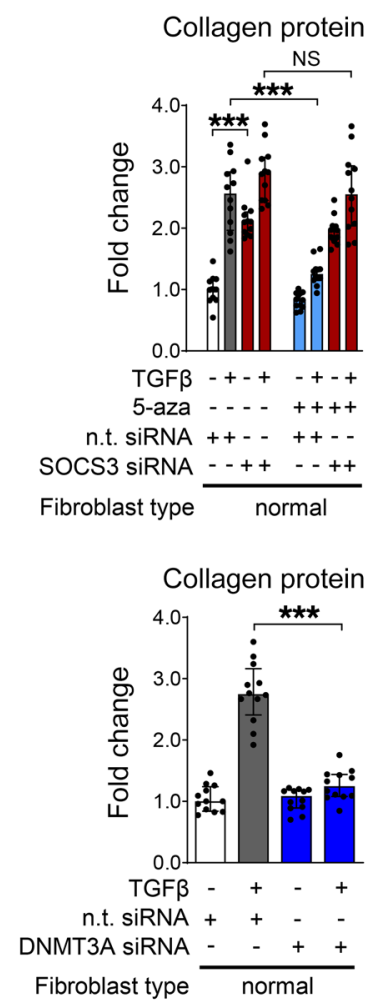

Figure 4. Knockdown of SOCS3 promotes fibroblast activation, while inactivation of DNMTs prevents it. (A) mRNA levels of COL1A1 and $C O L 1 A 2$ and collagen protein levels upon knockdown of SOCS3 in fibroblasts from healthy individuals stimulated with TCF- $\beta$ and incubated with 5 -aza. $n=6$ fibroblast lines from different donors with 2 technical replicates each for all conditions. (B) mRNA levels of COL1A1 and COL1A2 and collagen protein levels upon knockdown of DNMT3A in normal dermal fibroblasts stimulated with TCF- $\beta . n=6$ fibroblast lines from different donors with 2 technical replicates each. (C) mRNA levels of COL1A1 and COL1A2 and collagen content in cell culture media upon knockdown of SOCS3 in fibroblasts from SSc patients. $n=8$ fibroblast lines from different donors with 2 technical replicates each for all conditions. (D) mRNA levels of COL1A1 and COL1A2 and relative collagen content in cell culture media after knockdown of DNMT1 or DNMT3A in fibroblasts from SSc patients. $n=8$ fibroblast lines from different donors with 2 technical replicates each for all conditions. Data are depicted as the median with interquartile range. Each dot represents an individual result. One-way ANOVA with Tukey's range test as post hoc analysis was used for statistical analyses. ${ }^{* *} P<0.001$.

dermal thickening, myofibroblast counts, and collagen deposition upon challenge with bleomycin (Figure 7, A and B, and Supplemental Figure 6B) or TBRI ${ }^{\text {act-AAV }}$ (Figure 7, C and D) compared with Dnmt3at/ll mice. In agreement with a Dnmt3a-induced downregulation of Socs3, additional treatment of Dnmt3a $a^{\text {f/f }}$ Col6Cre mice with 5 -aza had no additive antifibrotic effects.

We also investigated whether individual knockdown of Dnmt1 or of Dnmt3a may induce regression of preestablished experimental skin fibrosis. Indeed, knockdown of Dnmt1 or Dnmt3a not only prevented progression of bleomycin-induced skin fibrosis, but reduced dermal thickness, myofibroblast counts, and hydroxyproline content to below pretreatment levels despite ongoing bleomycin challenge (Supplemental Figure 8).

SOCS3 inhibits JAK2/STAT3 signaling to regulate TGF- $\beta$-dependent fibroblast activation. SOCS3 can bind to JAK1, JAK2, and TYK2 to inhibit their kinase activity, preventing subsequent activation of STAT and transcription of STAT-dependent target genes $(37,38)$. We and others previously es in the texture of the fur, reduced activity, other abnormal behavior, or altered consistency of the stool in mice treated with 5-aza.

In contrast to the knockout of Socs3 in fibroblasts, mice with fibroblast-specific knockout of Dnmt3a (Dnmt3at/fl Col6Cre) were protected from experimental fibrosis and demonstrated reduced demonstrated that stimulation with TGF- $\beta$ activates JAK2/STAT3 signaling in fibroblasts, characterized JAK2 and STAT3 as important intracellular mediators of TGF- $\beta$ signaling, and demonstrated enhanced activation of JAK2/STAT3 signaling with accumulation of phosphorylated JAK2 (pJAK2) and phosphorylated STAT3 (pSTAT3) 

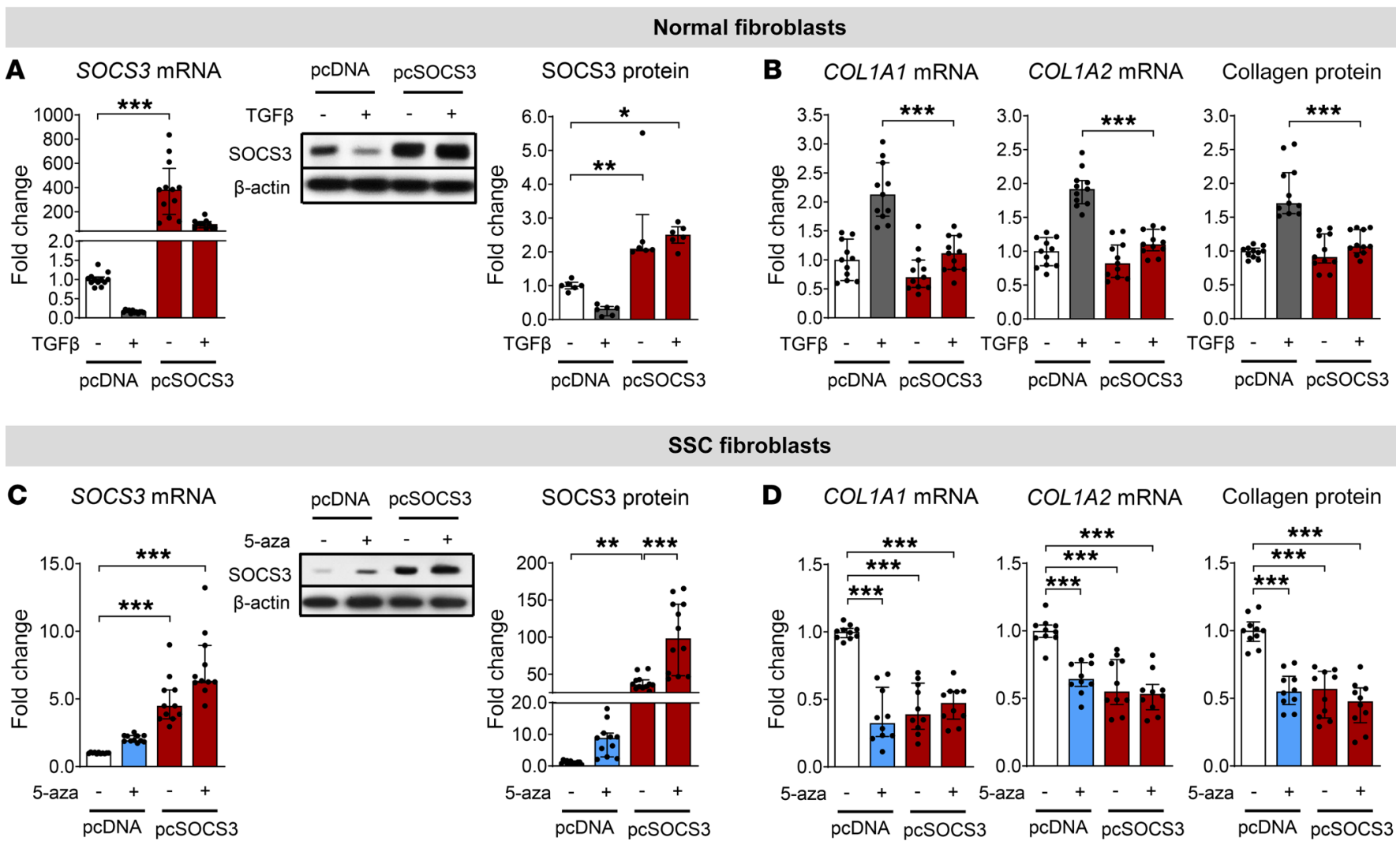

Figure 5. Overexpression of SOCS3 inhibits collagen release. (A and B) Overexpression of SOCS3 in dermal fibroblasts from healthy individuals. (A) mRNA and protein levels of SOCS3 upon forced overexpression of SOCS3 in fibroblasts from healthy individuals. $n=6$ fibroblast lines from different donors with 2 technical replicates each for qPCR; $n=3$ fibroblast lines from different donors with 2 technical replicates each for Western blot. (B) mRNA levels of COL1A1 and COL1A2 and relative collagen protein in cell culture supernatant upon overexpression of SOCS3 and stimulation with TGF- $\beta$. $n=6$ fibroblast lines from different donors with 2 technical replicates each. (C and D) Overexpression of SOCS3 in SSc fibroblasts. (C) mRNA and protein levels of SOCS3 upon forced overexpression of SOCS3 in fibroblasts from SSc patients. $n=6$ fibroblast lines from different donors with 2 technical replicates each for qPCR; $n=4$ fibroblast lines from different donors with 3 technical replicates each for Western blot. (D) COL1A1 and COL1A2 mRNA and relative collagen protein in cell culture supernatant. $n=5$ fibroblast lines from different donors with 2 technical replicates each. Data are depicted as the median with interquartile range. Each dot represents an individual result. One-way ANOVA with Tukey's range test as post hoc analysis was used for statistical analyses. $0.05>{ }^{*} P \geq 0.01 ; 0.01>{ }^{* *} P \geq 0.001 ;{ }^{* *} P<0.001$

in SSc (33-35, 60). We thus tested the hypothesis that SOCS3 may regulate fibroblast activation by inhibiting TGF- $\beta$-induced JAK2/ STAT3 signaling. Indeed, when we knocked down JAK2 in addition to SOCS3 in normal fibroblasts, we were able to completely rescue the activated phenotype of SOCS3 knockdown fibroblasts (Supplemental Figure 9). The mRNA levels of COL1A1 and of COL1A2 and the release of collagen protein were reduced in fibroblasts with combined knockdown of JAK2 and SOCS3 compared with fibroblasts with individual knockdown of SOCS3 and were similar to those of fibroblasts with individual knockdown of JAK2 (Supplemental Figure 9A). In addition, coknockdown of JAK2 in addition to SOCS3 reduced the activation of fibroblasts with decreases in $\alpha$-SMA expression and stress fiber formation (Supplemental Figure 9B).

Given the regulation of SOCS3 by promoter hypermethylation, inhibition of the epigenetic silencing of SOCS3 should ameliorate TGF- $\beta$-induced JAK2/STAT3 signaling. Indeed, coincubation of normal dermal fibroblasts with 5-aza prevented the accumulation of pSTAT3 upon prolonged stimulation with TGF- $\beta$, whereas knockdown of SOCS3 promoted it (Supplemental Figure 9C). Co-IP further demonstrated that coincubation of TGF- $\beta$-stimulated fibro- blasts with 5 -aza restored the negative feedback regulation by SOCS3 with increased binding of SOCS3 to JAK2 and decreased interaction of JAK2 with STAT3 (Figure 8A). Consistently, coincubation with 5 -aza inhibited the stimulatory effects of TGF- $\beta$ on STAT3-regulated transcription in reporter assays (Figure $8 \mathrm{~B}$ ). The inhibitory effects of 5-aza on STAT3-dependent transcription were absent in SOCS3 knockdown fibroblasts. In contrast, knockdown of DNMT3A significantly decreased responsiveness to TGF- $\beta$ and reduced STAT3 reporter activity (Figure $8 \mathrm{~B}$ ). Forced overexpression of SOCS3 inhibited STAT3-dependent transcriptional activity in these assays, and the effect of forced overexpression of SOCS3 was not sensitive to 5-aza (data not shown).

To further demonstrate that the activated phenotype of SOCS3 knockdown fibroblasts is caused by hyperactivation of JAK2/STAT3 signaling, we overexpressed a mutant version of JAK2 with point mutations in the GQM-binding motif that is required for binding of SOCS3 (JAK2-G1071V;M1073A) (Supplemental Figure 10) (36). Incubation with 5-aza upon forced overexpression of WT JAK2 (JAK2-WT) diminished the TGF- $\beta$-induced increases in pSTAT3 levels, collagen and $\alpha$-SMA expression, and stress fiber formation (Figure 8, C-E). How- 
A

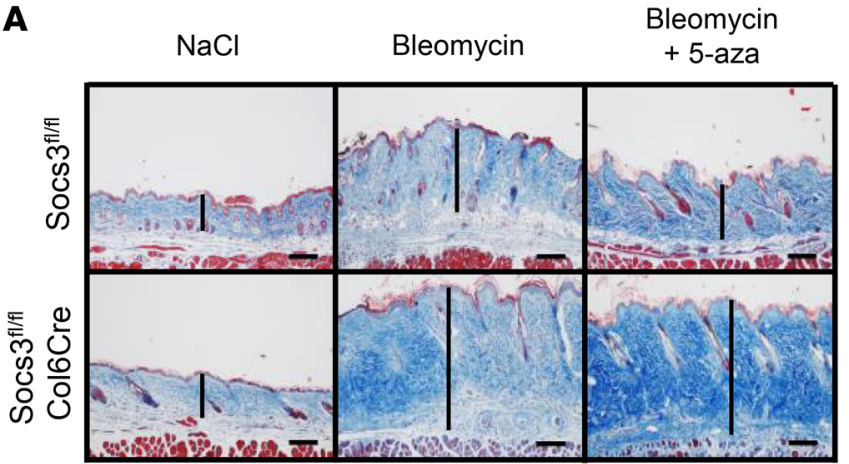

C

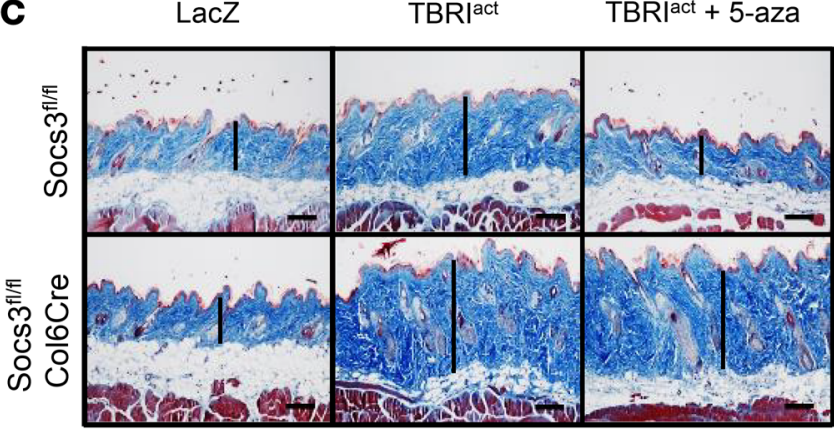

B

Dermal thickness Myofibroblast counts Hydroxyproline

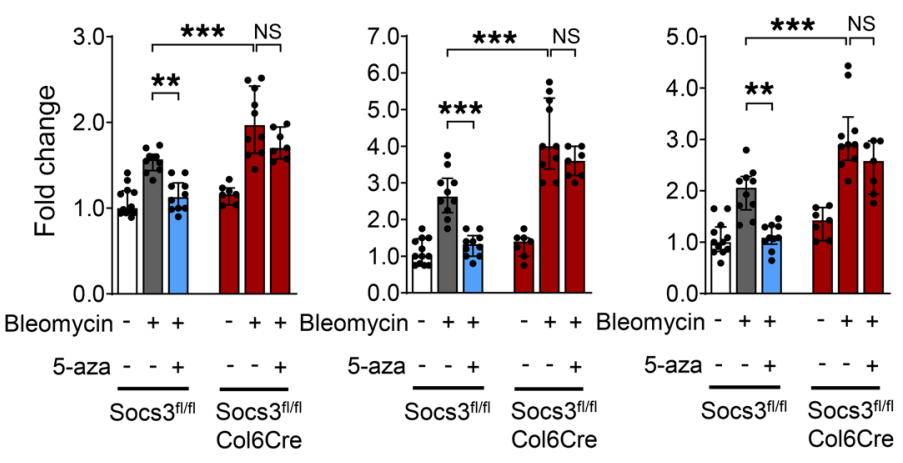

D Dermal thickness

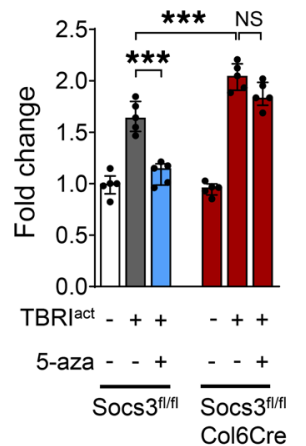

Myofibroblast counts

Hydroxyproline

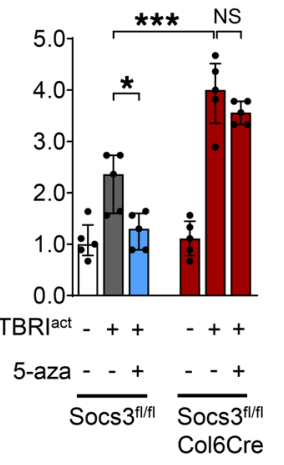

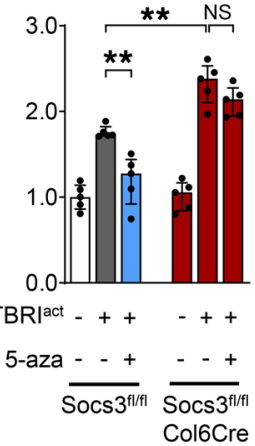

Figure 6. Fibroblast-specific knockout of Socs3 exacerbates experimental fibrosis. (A and B) Bleomycin-induced dermal fibrosis. (A) Representative trichrome-stained skin sections. Original magnification, $\times 100$. Scale bars: $250 \mu \mathrm{m}$. (B) Quantitation of dermal thickness, myofibroblast counts, and hydroxyproline content. $n \geq 7$ mice per group. (C and D) TBRIact-induced dermal fibrosis. (C) Representative trichrome-stained skin sections. Original magnification, $\times 100$. Scale bars: $250 \mu \mathrm{m}$. (D) Quantitation of dermal thickness, myofibroblast counts, and hydroxyproline content. $n=5$ mice per group. Data are depicted as the median with interquartile range. Each dot represents an individual result. One-way ANOVA with Tukey's range test as post hoc analysis was used for statistical analyses. $0.05>{ }^{*} P \geq 0.01 ; 0.01{ }^{* *} P \geq 0.001 ;{ }^{* *} P<0.001$.

ever, the antifibrotic effects of 5-aza were blunted in JAK2-G1071V;$M 1073 A$ fibroblasts. In contrast to what occurred with controls, 5-aza did not change the levels of pSTAT3, collagen, and the expression of myofibroblast markers in TGF- $\beta$-stimulated JAK2-G1071V;M1073A fibroblasts (Figure 8, C-E). Together, these data demonstrate that the antifibrotic effects of 5-aza are mainly mediated by SOCS3-regulated inhibition of JAK2/STAT3 signaling.

To confirm these findings in cultured fibroblasts in the context of fibrosis, we analyzed the effects of knockdown of Socs 3 and Dnmt3a and of treatment with 5-aza on the activation of Jak2/Stat3 signaling in experimental fibrosis. Immunofluorescence staining of pJak2 and pStat 3 in the dermis of bleomycin- and TBRI act-induced fibrosis models showed that inhibition of DNA methylation by 5 -aza effectively reduced Jak2/Stat3 signaling in experimental fibrosis (Supplemental Figures 11-14). However, such inhibitory effects of 5 -aza on Jak2/Stat3 activation were not observed in fibroblast-specific Socs3 mutants (Socs $3^{\text {f/fl}}$ Col6Cre mice), indicating that Socs3 essentially regulates Jak2/Stat3 signaling. (Supplemental Figures 11 and 12). Conversely, when investigating fibroblast-specific Dnmt3a mutants (Dnmt3at/ll Col6Cre mice), which are associated with high-level Socs3 expression, bleomycin and TBRI ${ }^{\text {act }}$ did not achieve Jak2 and Stat3 activation (Supplemental Figures 13 and 14). Also, 5-aza had no further effects on Jak2 and Stat3 activation.

\section{Discussion}

In the present study, we demonstrate that the expression of SOCS3 is reduced in fibroblasts of the fibrotic skin of SSc patients, a phenotype that persists even in isolated fibroblasts after longterm in vitro culture. The expression of Socs 3 is also decreased in murine models of skin fibrosis. We show on multiple experimental levels that canonical TGF- $\beta$ signaling plays an important role for the downregulation of SOCS3: (a) incubation with recombinant TGF- $\beta$ decreased the mRNA and protein levels of SOCS3 in cultured fibroblasts; (b) selective activation of TGF- $\beta$ signaling by overexpression of TBRI ${ }^{\text {act }}$ reduced the expression of Socs 3 in murine skin; and (c) treatment with SD-208, a selective inhibitor of TGF- $\beta$ receptor type I (61), prevented the downregulation of Socs 3 in experimental fibrosis. Each of these findings demonstrates that TGF- $\beta$ signaling is required to downregulate SOCS3 in fibrosis. However, in addition, other factors relevant to the pathogenesis of fibrosis and SSc, such as hypoxia, may regulate the expression of SOCS3 and hence may contribute to the SOCS3 decrease in patients with $\mathrm{SSc}(50,62)$.

Mechanistically, TGF- $\beta$ reduces the expression of SOCS3 by induction of DNMT3A, which silences SOCS3 expression by DNA methylation. We demonstrate by methylation-specific PCR and MeDIP assays that the promoter of SOCS3 is hypermethylated in 
A

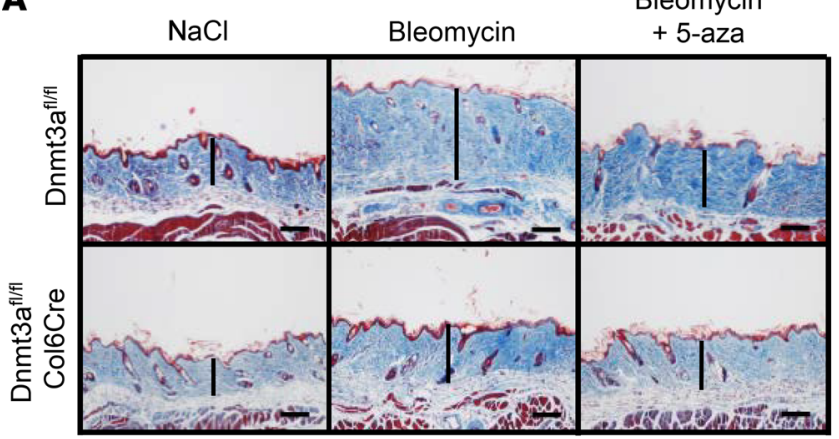

C

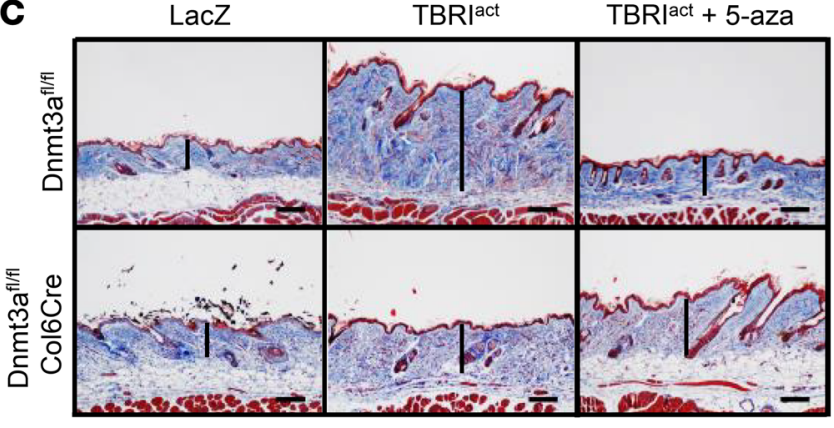

B Dermal thickness

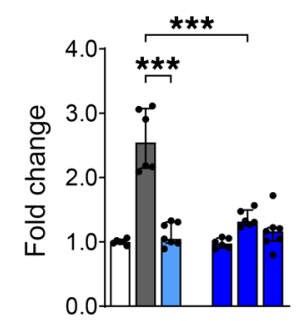

Bleomycin - +

5-aza - - + $\begin{array}{cc}\overline{\text { Dnmt3afl/fl }} & \overline{D n m t 3} a^{f / f f l} \\ \text { Col6Cre }\end{array}$

D Dermal thickness

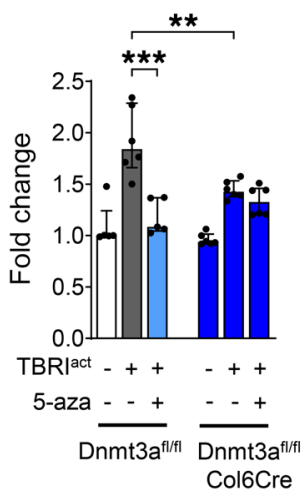

Myofibroblast counts

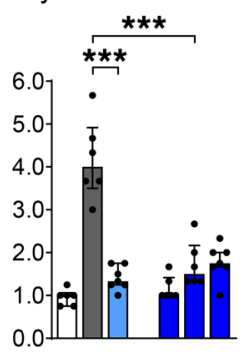

mycin - +

- + Bleom

5-aza - -+

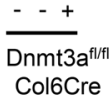

Myofibroblast counts

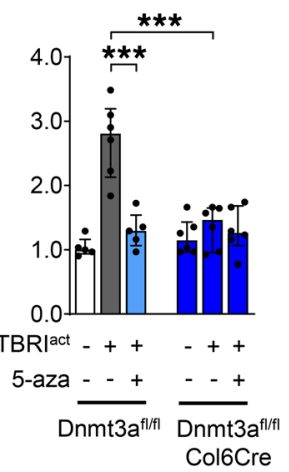

Hydroxyproline

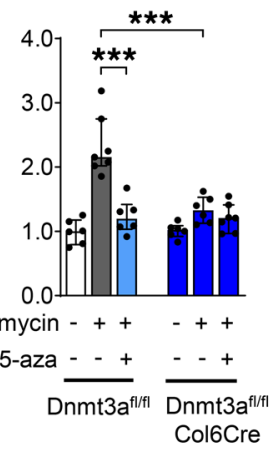

Hydroxyproline

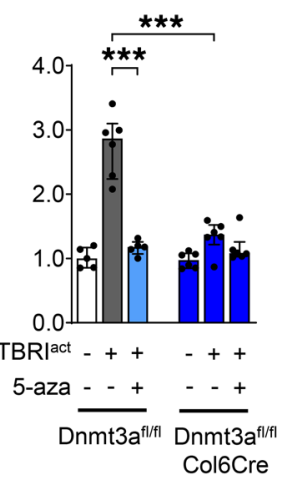

Figure 7. Fibroblast-specific knockout of Dnmt3a ameliorates experimental fibrosis. (A and B) Bleomycin-induced dermal fibrosis. (A) Representative trichrome-stained skin sections. Original magnification, $\times 100$. Scale bars: $250 \mu \mathrm{m}$. (B) Quantitation of dermal thickness, myofibroblast counts, and hydroxyproline content. $n=7$ mice per group. (C and D) TBRact-induced dermal fibrosis. (C) Representative trichrome-stained skin sections. Original magnification, $\times 100$. Scale bars: $250 \mu \mathrm{m}$. (D) Quantitation of dermal thickness, myofibroblast counts, and hydroxyproline content. $n=5$ mice per group. Data are depicted as the median with interquartile range. Each dot represents an individual result. One-way ANOVA with Tukey's range test as post hoc analysis was used for statistical analyses. $0.01>^{* *} P \geq 0.001 ;{ }^{* *} P<0.001$

SSc fibroblasts compared with fibroblasts from healthy individuals. Stimulation of normal fibroblasts with recombinant TGF- $\beta$ not only leads to transformation into an SSc-like fibroblast phenotype, but also upregulates methylation of the SOCS3 promoter to levels comparable to those observed in SSc fibroblasts. The downregulation of SOCS3 in SSc fibroblasts is thus consistent with the persistent activation of TGF- $\beta$ signaling in these cells even after several passages in vitro (4). The stimulatory effects of TGF- $\beta$ on SOCS3 promoter methylation are mediated by a SMAD-dependent induction of DNMT3A. Prolonged incubation with TGF- $\beta$ also induced the expression of DNMT1. However, the kinetic profiles of DNMT3A and DNMT1 upregulation suggest that DNMT3A is induced directly and operates as de novo DNMT at the SOCS3 promoter, whereas DNMT1 is induced indirectly and rather serves to maintain the preexisting DNA methylation marks (21). According to this model, the activity of both DNMTs would be required to chronically sustain promoter hypermethylation of SOCS3 and inactivation of either DNMT1 or DNMT3A would prevent chronic silencing of SOCS3 and other target genes $(24,63)$. Consistently, we found that, in addition to the pronounced upregulation of DNMT3A, the expression of DNMT1 was also increased in SSc.

Previous elegant studies demonstrated that the protective antifibrotic transcription factors FLI1 and KLF5 are silenced by
DNA hypermethylation in SSc and that their silencing is directly implicated into different aspects of the pathogenesis of SSc (29, $30,64)$. Our study demonstrates, using the example of SOCS3, that the aberrant activation of DNMTs in SSc can also promote activation of profibrotic transcriptional programs. Inactivation of SOCS3 lowers the threshold for STAT3 activation and results in prolonged activation of STAT3, which may directly contribute to the autonomous activation of SSc fibroblasts. Silencing of SOCS3 amplified the responsiveness of fibroblasts to TGF- $\beta$, and fibroblast-specific deletion of Socs3 in vivo worsened skin fibrosis. In contrast, forced expression of SOCS3, knockout of DNMT3A, or treatment with 5-aza inhibited TGF- $\beta$-induced myofibroblast differentiation, collagen production, and skin fibrosis. Induction of DNMT3A with subsequent silencing of SOCS3 thus directly contributes to the profibrotic effects of TGF- $\beta$.

These findings may have therapeutic implications. They highlight a central role of STAT3 in the pathogenesis of SSc and demonstrate that activation of STAT3 signaling is sufficient to promote fibroblast activation and contributes to the activated phenotype of SSc fibroblasts. Reestablishment of the endogenous feedback regulation of STAT signaling either by forced reexpression of SOCS3 or by inhibition of DNA methylation by targeting DNMT3A reduces the endogenous activation of SSc fibroblasts, limits the profi- 
A

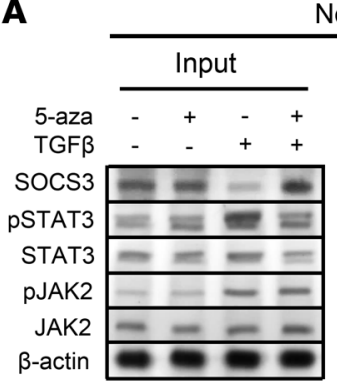

B

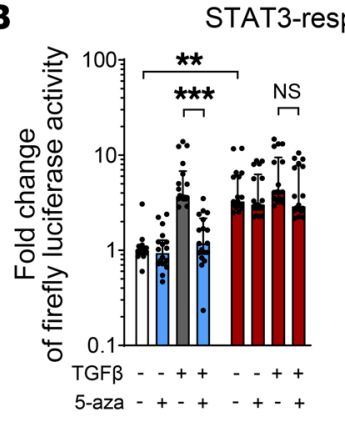

Normal fibroblasts

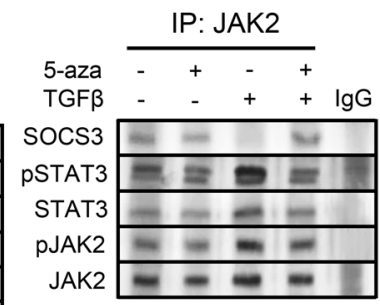

IP JAK2:

SOCS3 protein

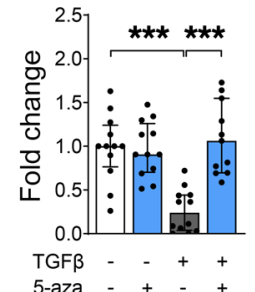

IP JAK2

pSTAT3 / STAT3 ratio

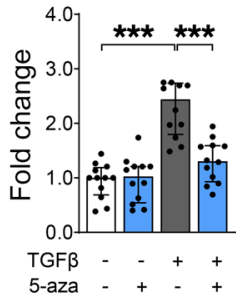

pSTAT3 / STAT3 ratio
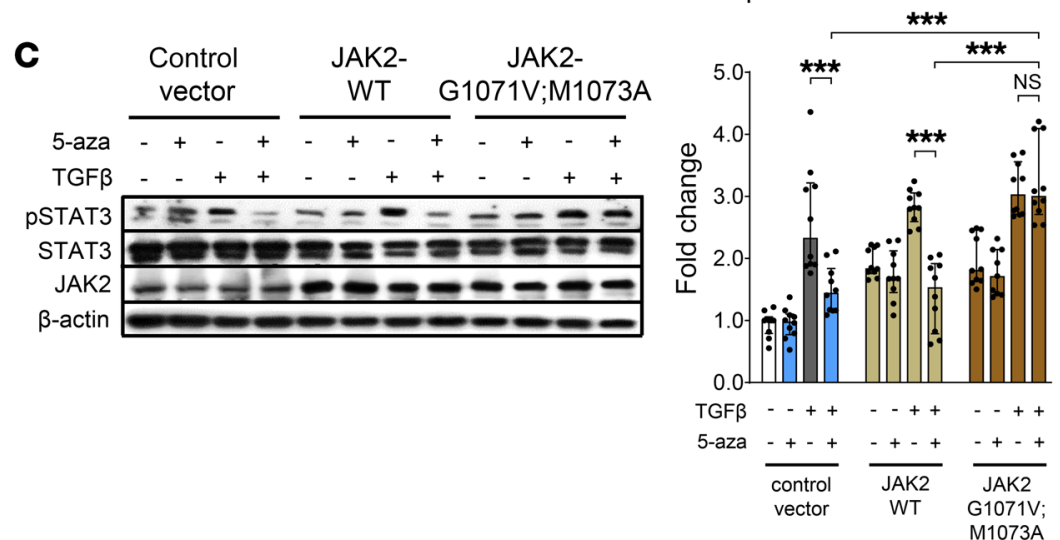

D

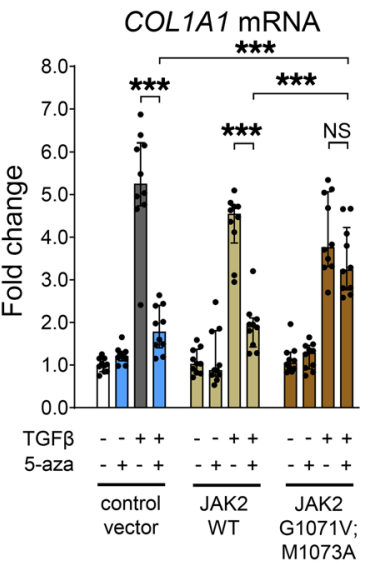

Collagen protein

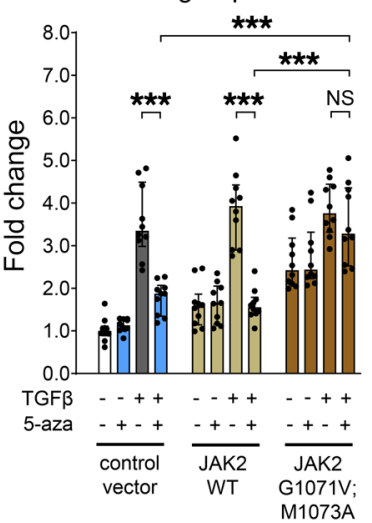

E

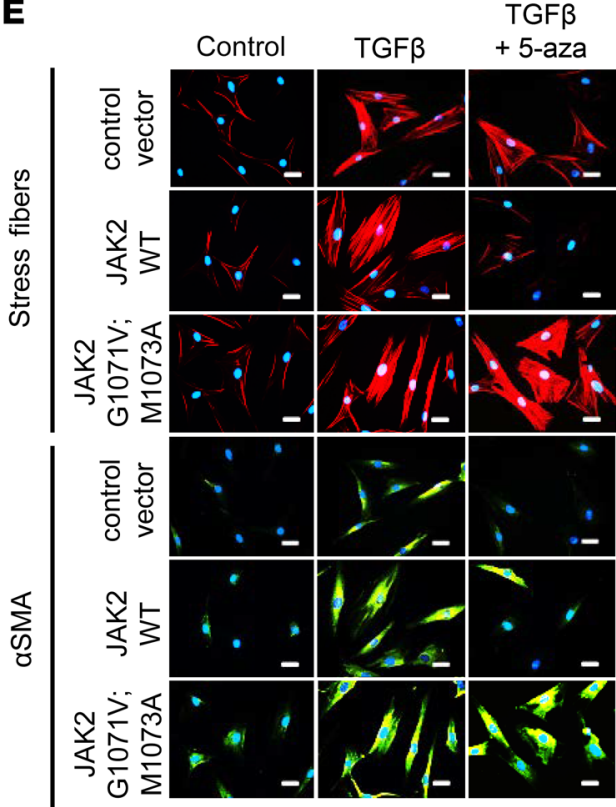

Stress fibers

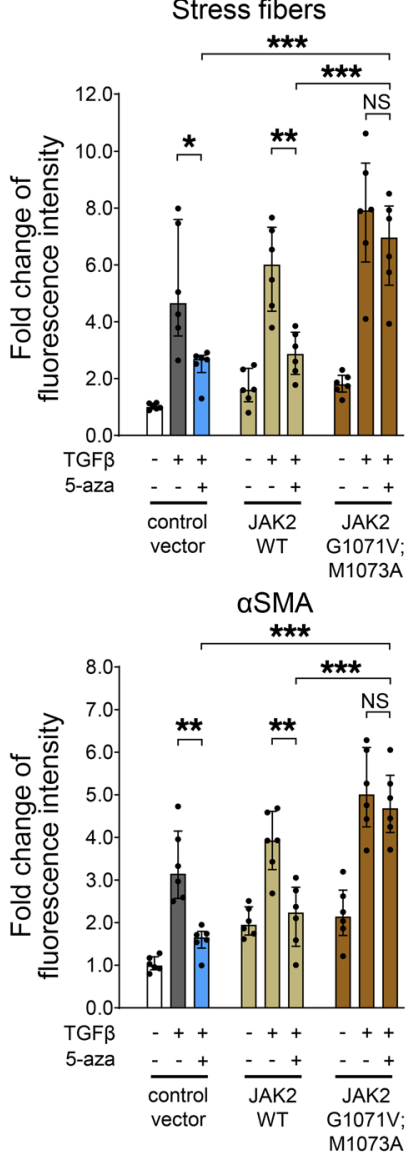


Figure 8. Mutation of the SOCS3-binding motif of JAK2 abrogates the beneficial effects of 5 -aza on TGF- $\beta$-induced collagen synthesis and myofibroblast differentiation. (A) Representative Western blot and quantitation of co-IP of JAK2, STAT3, and SOCS3 in normal dermal fibroblasts stimulated with TCF- $\beta$ and incubated with 5 -aza. $n=4$ fibroblast lines from different donors with 3 technical replicates each. (B) Effects of 5-aza and of siRNA-mediated knockdown of SOCS3 (left) or DNMT3A (right) on TCF- $\beta$-induced firefly luciferase activity under the control of a STAT3responsive promoter. $n=6$ fibroblast lines from different donors with 3 technical replicates each. (C) Representative Western blot and quantitation of the PSTAT3/STAT3 ratio upon overexpression of WT JAK2 (JAK2-WT) or mutated JAK2 with a defective SOCS3-binding site (JAK2-G1071V;M1073A) in normal dermal fibroblasts stimulated with TGF- $\beta$ and incubated with 5 -aza. $n=4$ fibroblast lines from different donors with 3 technical replicates each. (D) COL1A1 mRNA and collagen protein levels in cell culture media upon overexpression of WT JAK2 or mutated JAK2 ( $n=5$ fibroblast lines from different donors with 2 technical replicates each). (E) Representative stainings and quantitations of $\alpha$-SMA (green) and stress fibers (red) upon overexpression of WT or mutated JAK2 in normal fibroblasts. $n=3$ fibroblast lines from different donors with 2 technical replicates each. Original magnification, $\times 400$. Scale bars: $100 \mu \mathrm{m}$. Data are depicted as the median with interquartile range. Each dot represents an individual result. One-way ANOVA with Tukey's range test as post hoc analysis was used for statistical analyses. $0.05>{ }^{*} P \geq 0.01 ; 0.01>{ }^{* *} P \geq 0.001 ;{ }^{* *} P<0.001$.

brotic effects of TGF- $\beta$, and ameliorates dermal fibrosis. Of note, reactivation of SOCS3 expression did not affect collagen release of resting fibroblasts from healthy individuals, suggesting that this approach may not significantly interfere with homeostatic functions of fibroblasts. More than 10 different clinical trials investigating STAT3 inhibitors in various tumors are currently ongoing or have been recently completed (e.g., ClinicalTrials.gov NCT01563302, NCT01663571, NCT01904123, NCT03195699), highlighting that STAT3 is considered as a prime target for pharmaceutical intervention and that numerous compounds would be available for clinical trials in SSc.

Our data also strengthen the scientific rationale for targeting DNA methylation in fibrotic diseases. Deletion of Dnmt3a or Dnmt1 or pharmacologic inhibition of Dnmts ameliorated fibrosis induced by bleomycin or by TBRI ${ }^{\text {act. }}$. Of note, treatment with 5-aza was not only effective in preventive, but also in therapeutic, settings and not only inhibited further progression, but also induced regression of preestablished fibrosis. Inhibition of DNMTs would not only limit aberrant STAT3 signaling in SSc, but would also exert antifibrotic effects by reactivation of protective genes such as FLI1 and $\operatorname{KLF5}(29,30)$.

However, the simultaneous modulation of several pathways by epigenetic modifiers may not only add to the therapeutic effects, but may also carry a higher risk of adverse events, as it may also interfere with pathways that are not relevant to the pathogenesis of SSc. However, these concerns may be limited by recent findings that treatment with 5-aza does not cause random DNA demethylation (65). Instead, treatment with 5-aza predominantly modulated the expression of a panel of regulatory genes, while it had little effects on the methylation status of homeostatic genes. In addition to potential effects on fibrotic manifestations, accumulating evidence suggests that aberrant DNA methylation may also be implicated in vascular and inflammatory manifestations of SSc and other rheumatic diseases $(20,66,67)$. Inhibition of DNA methylation may thus offer unique potential to improve a broad spectrum of manifestations of SSc. However, further studies in additional preclinical models that better resemble vascular and inflammatory manifestations of SSc are warranted to confirm this hypothesis.

In summary, we demonstrate that chronic activation of TGF- $\beta$ signaling perturbs the epigenetic control of STAT signaling by DNMT3A-induced silencing of SOCS3 expression (Figure 9). Reestablishment of the endogenous regulation of STAT signaling, either by forced expression of SOCS3 or by inhibition of DNMTs, prevents aberrant STAT3 signaling, inhibits TGF- $\beta$-induced fibroblast activation and collagen release, and ameliorates experimental fibrosis. Restoration of the epigenetic control of STAT3 signaling might thus be a novel approach for the treatment of fibrotic diseases such as SSc.

\section{Methods}

Patients and fibroblast cultures. Skin biopsies were obtained from the forearms of $38 \mathrm{SSc}$ patients and 42 age- and sex-matched healthy volunteers. All patients fulfilled the American College of Rheumatology (ACR)/European League Against Rheumatism (EULAR) criteria for SSc (68). Twenty-two patients had diffuse cutaneous SSc and 16 had limited cutaneous SSc. The median age of SSc patients was 46 years (range: 19-70 years), and their median disease duration was 5 years (range: 0.5-12 years). All patients were positive for anti-nuclear Abs; 14 patients were positive for anti-topoisomerase-1 Abs, and 12 were positive for anti-centromere Abs, 2 for anti-RNAIII polymerase Abs. Patients did not receive any disease-modifying anti-rheumatic drug treatment at the time of biopsy.

Cell culture. Fibroblasts were prepared by outgrowth of skin biopsies and cultured as described (69-71). Dermal fibroblasts were stimulated with recombinant TGF- $\beta(10 \mathrm{ng} / \mathrm{mL}$; Peprotech) for different time intervals up to 120 hours. For inhibition of DNMTs, cells were incubated with 5-aza (Sigma-Aldrich) in concentrations of $1 \mu \mathrm{M}$ and $10 \mu \mathrm{M}$ for different time intervals. In a subset of experiments, recombinant TGF- $\beta$ was added 60 minutes after 5 -aza. All experiments were performed with fully confluent cells. In a subset of experiments, fibroblasts were incubated with the following cell cycle inhibitors: SKPinC1 at $0.5 \mu \mathrm{M}$ (Tocris, Bio-Techne GmbH), ABT751 at $0.2 \mu \mathrm{M}$, and AZD5438 at $0.2 \mu \mathrm{M}$ (both Selleckchem) to exclude confounding effects of cell proliferation.

RNAi and overexpression experiments. Dermal fibroblasts were transfected with $3 \mu \mathrm{g}$ of siRNA duplexes against SOCS3, JAK2, DNMT1, DNMT3A, SMAD3, or SMAD4 using the 4D-Nucleofector Kit for human dermal fibroblasts (Lonza Cologne $\mathrm{GmbH}$ ), as previously described $(70,72)$. The sequences of the siRNAs are summarized in Supplemental Table 1. Fibroblasts transfected with nontargeting siRNAs (Eurogentec) served as controls. In order to overexpress SOCS3 in dermal fibroblasts, the cDNA of SOCS3 was cloned into pcDNA3.1(+) expression vectors (Thermo Fisher Scientific).

The plasmid pGEM-JAK2 encoding the human JAK2 gene was purchased from Sino Biological. In vitro mutagenesis of the GQM-binding motif for SOCS3 of JAK2 was performed using the QuikChange Multi Site-Directed Mutagenesis Kit (Agilent Technologies) to obtain the mutant JAK2-G1071V;M1073A, which cannot be inhibited by SOCS3 (36). After verification of the correct sequence, the constructs were transfected in dermal human fibroblasts using the Lonza 4D-Nucleofector Kit. Fibroblasts were transfected with $5 \mu \mathrm{g}$ of plasmids (73). Transfections with the empty or nonmutated WT vectors were used as controls. 
A Physiological response with temporary upregulation of TGF $\beta$

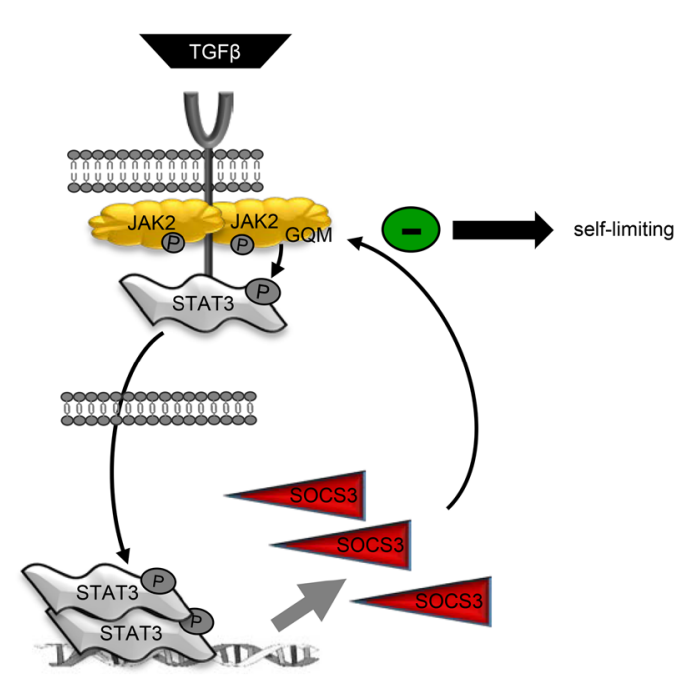

B Fibrotic diseases with persistent upregulation of TGF $\beta$

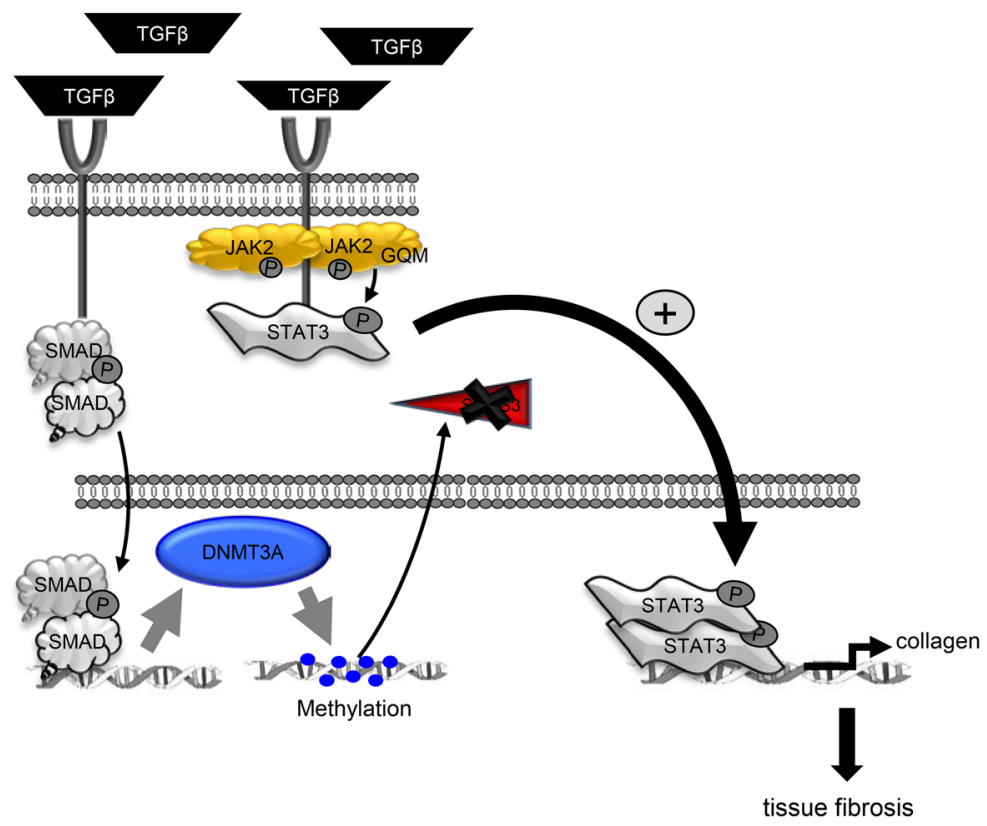

Figure 9. Graphical summary of the proposed mechanism. (A) Physiological response with temporary upregulation of TCF- $\beta$. (B) Mechanism during fibrotic tissue remodeling with persistent activation of TCF- $\beta$ signaling.

Methylation-specific PCR and MeDIP. Genomic DNA was isolated from fibroblasts of SSc patients and healthy volunteers using the QIAGEN DNeasy Extraction System (QIAGEN) according to the manufacturer's instruction.

For methylation-specific PCR, $2 \mu \mathrm{g}$ of genomic DNA were processed using the EpiTect Bisulfite Kit (QIAGEN). The sequences of the primer pairs used for methylation- and unmethylation-specific PCR are summarized in Supplemental Table 2. The reaction mixture contained $50 \mathrm{ng}$ bisulfite converted DNA in a final volume of $50 \mu \mathrm{l}$. Each product was loaded onto a $2 \%$ agarose gel and visualized under ultraviolet illumination. Each band was quantified using Image J software (NIH, version 1.42).

For MeDIP assay, genomic DNA was fractionated by enzymatic digestion with BmrI and EcoP15I (New England Biolabs) and processed for immunoprecipitation using the MeDIP Assay Kit from Active Motif according to the manufacturer's protocol. The primer sequences used for qPCR are given in Supplemental Table 3.

ChIP. ChIP assays were performed using the ChIP-IT Express Kit (Active Motif) as described (74). Anti-SMAD3 Abs (Smad3 [C67H9] rabbit mAb, catalog 9523, Cell Signaling Technology) and rabbit IgG Abs (catalog sc-2028 [discontinued], Santa Cruz Biotechnologies) were used. The primer sequences used for qPCR are given in Supplemental Table 3.

DNMT activity assay. DNMT activity was determined using a DNMT Activity/Inhibition Assay Kit (Active Motif) according to the manufacturer's protocol. Briefly, nuclear extracts were isolated with the nuclear extract kit (Active Motif), and $1.5 \mu \mathrm{g}$ of nuclear protein was incubated with $\mathrm{CpG}$-enriched DNA substrate immobilized on the reaction plates for 2.5 hours at $37^{\circ} \mathrm{C}$. The absorbance was analyzed at $450 \mathrm{~nm}$ with $655 \mathrm{~nm}$ as reference wavelength with a Spectra MAX 190 microplate spectrophotometer (Molecular Devices).
Reporter assay. Fibroblasts were transfected with $1 \mu \mathrm{g}$ of STAT3-luciferase reporter construct (QIAGEN) in serum-free medium using the Lonza 4D-Nucleofector Kit (73). Transfected cells were incubated with TGF- $\beta$ and 5-aza for 16 hours and harvested 36 hours after transfection. A noninducible luciferase vector was used as control. Luciferase activity was determined using a microplate luminometer (Berthold Technologies).

Quantitative real-time PCR. Total RNA was isolated with the NucleoSpin RNA II extraction system (MACHEREY-NAGEL) and reverse transcribed into cDNA as described $(75,76)$. Gene expression was quantified by real-time PCR using the Mx3005P Sequence Detection System (Agilent Technologies). Specific primer pairs for each gene were designed with Primer 3 software. The primer sequences used are given in Supplemental Table 3. Measurement of $\beta$-actin levels was used to normalize for the amounts of loaded cDNA. Samples without enzyme in the reverse transcription reaction, without template and dissociation curve analysis, served as controls. Differences were calculated with $\mathrm{Ct}$ and the comparative $\mathrm{Ct}$ method for relative quantification.

Western blot. Protein samples were separated by SDS-polyacrylamide gel and electrotransferred onto polyvinylidene fluoride (PVDF) membranes (Merck Millipore). After blocking, membranes were incubated with mouse anti-SOCS3 (clone SOC-25, catalog 626601, BioLegend), rabbit anti-SOCS3 (polyclonal, catalog ab16030, Abcam), rabbit anti-DNMT1 (polyclonal, catalog PLA0011, Sigma-Aldrich), rabbit anti-DNMT3A (polyclonal, catalog ab2850, Abcam), mouse anti-DNMT3B (clone 52A1018, catalog NB100-56514, Novus Biologicals), rabbit anti-SMAD3 (clone C67H9, catalog 9523, Cell Signaling Technology), rabbit anti-SMAD4 (polyclonal, catalog 9515, Cell Signaling Technology), rabbit anti-JAK2 (clone D2E12, catalog 3230, Cell Signaling Technology), rabbit anti-phospho-JAK2 (Tyr1007/1008) (polyclonal, catalog 3771, Cell Signaling Technology), mouse anti- 
STAT3 (clone 124H6, catalog 9139, Cell Signaling Technology), or rabbit anti-phospho-STAT3 (Tyr705) (clone D3A7, catalog 9145, Cell Signaling Technology) overnight at $4^{\circ} \mathrm{C}$. Equal loading of proteins was confirmed by incubation with mouse anti- $\beta$-actin (clone AC- 15 , catalog A5441, Sigma-Aldrich). Membranes were incubated with horseradish peroxidase-conjugated secondary Abs (Agilent Technologies).

Immunohistochemical and immunofluorescence staining. Paraffin-embedded skin sections or cultured fibroblasts were stained with mouse anti-P4H $\beta$ (clone RL90, catalog MA3-019, Thermo Fisher Scientific), rhodamine-conjugated phalloidin (Thermo Fisher Scientific), mouse anti- $\alpha$-SMA (clone ASM-1/1A4, catalog CBL171-I, Sigma-Aldrich), mouse anti-vimentin (clone VI-10, catalog ab20346, Abcam), rabbit anti-SOCS3 (polyclonal, catalog ab16030, Abcam), rabbit anti-DNMT3A (polyclonal, catalog ab2850, Abcam), goat anti-phospho-JAK2 (Tyr1007/1008) (polyclonal, catalog sc-21870, Santa Cruz Biotechnology), goat anti-phospho-STAT3 (Tyr705) (polyclonal, sc-7993, Santa Cruz Biotechnology), and DAPI (CAS 28718-90-3, catalog sc-3598, Santa Cruz Biotechnology). Concentration-matched species-specific immunoglobulins (Thermo Fisher Scientific) served as control Abs. Stainings were analyzed using a Nikon Eclipse 80i microscope.

Co-IP. Fibroblasts were collected in lysis buffer $(20 \mathrm{mM}$ Tris- $\mathrm{HCl}$ pH 8, $137 \mathrm{mM} \mathrm{NaCl}, 10 \%$ glycerol, $1 \%$ NP-40, and 2 mM EDTA). Cell extracts were incubated with rabbit anti-JAK2 (clone D2E12, catalog 3230, Cell Signaling Technology) or normal rabbit IgG Abs (Vector Laboratories) and $20 \mu \mathrm{L}$ of protein A/G Agarose (Santa Cruz Biotechnology). Unbound proteins were removed by washing with PBS. Agarose-bound protein complexes were separated via SDS-PAGE followed by Western blotting on a PVDF membrane. Ten percent of the lysates were used as input.

Collagen measurements. Total soluble collagen in cell culture supernatants was quantified using the SirCol collagen assay (Biocolor), as described (75). The absorbance was determined at $540 \mathrm{~nm}$ with a Spectra MAX 190 microplate spectrophotometer (Molecular Devices).

Murine models of fibrosis. Fibrosis was induced by subcutaneous injections of bleomycin every other day as described $(77,78)$. In order to analyze the efficiency of 5-aza for treatment of established fibrosis, the mouse model of preestablished bleomycin-induced dermal fibrosis was used in 6-week-old female DBA/2 mice (Janvier Labs) (79). One group receiving bleomycin for 6 weeks was additionally treated with intraperitoneal injections of 5 -aza for the last 3 weeks at a dose of $0.5 \mathrm{mg} / \mathrm{kg}$ every third day.

In addition to the model of bleomycin-induced dermal fibrosis, we also used the model of dermal fibrosis induced by local overexpression of TBRI act, as previously described $(72,77)$. Briefly, mice were injected with $6.67 \times 10^{7}$ plaque-forming units of TBRI ${ }^{\text {act }}-\mathrm{AAV} 5$ every other week. Control mice were injected with the same amounts of LacZ-encoding AAV5. Mice were sacrificed after 8 weeks for further analyses.

Fibrotic changes were analyzed by quantification of the dermal thickness, the number of $\alpha$-SMA-positive myofibroblasts, and hydroxyproline content $(72,80-82)$. Trichrome staining was performed for direct visualization of collagen.

Conditional knockout of Socs 3 and Dnmt3a. Mice with conditional alleles of Socs3 $\left(\right.$ Socs $\left.3^{f / f l}\right)(83)$ or Dnmt3a (Dnmt3a $\left.a^{f / f t}\right)(84)$ were crossbred with Col6-Cre mice (85) to obtain mice with selective knockout of Socs3 or Dnmt3a in fibroblasts $\left(\right.$ Socs $3^{f / f l}$ Col6Cre or Dnmt $3 a^{f / f l} \mathrm{Col}$ $6 \mathrm{Cre})$. Socs $3^{f / f l}$ mice and $\mathrm{Dnmt} 3 \mathrm{a}^{f / f l}$ mice were provided in-house, and Col6Cre mice were obtained from G. Kollias (Institute of Immunology,
Biomedical Sciences Research Center [BSRC] "Alexander Fleming," Vari, Greece). Six groups each were analyzed: 3 groups consisted of Socs $3^{f / f l}$ or Dnmt $3 a^{f / f l}$ mice; the other 3 groups consisted of Socs $3^{f / f l}$ Col6Cre or Dnmt $3 a^{f / f l}$ Col6Cre mice. Two of these 3 groups received bleomycin injections for 4 weeks or injections of TBRI ${ }^{\text {act-AAV5, as }}$ described above. One of the 2 groups was additionally treated with 5 -aza at a dose of $0.5 \mathrm{mg} / \mathrm{kg}$ every third day. The third group served as a control group and received injections with $0.9 \% \mathrm{NaCl}$ or LacZ-AAV5.

siRNA-mediated knockdown of Dnmt1 and Dnmt3a in murine skin. Female DBA/2 mice 6 weeks of age were purchased from Janvier Labs. Complexes of siRNA and atelocollagen (Koken) were prepared as described previously $(86,87)$. The siRNA sequences (Dharmacon, Horizon Discovery) are summarized in Supplemental Table 1. Nontargeting siRNA duplexes served as controls. Atelocollagen/siRNA complexes were injected intracutaneously once weekly.

Statistics. Results were visualized and analyzed with GraphPad Prism, version 8.1.2, and are depicted as the median with interquartile range. Dots represent individual values. For a 2-group comparison, Mann-Whitney $U$ test for nonparametric data was used. When more than 2 groups of samples were compared, 1-way ANOVA with Tukey's range test as post hoc analysis was used. A $P$ value of less than 0.05 was considered significant.

Study approval. The study was approved by the local institutional review boards (Ethikkommission of the Friedrich-Alexander-Universität Erlangen-Nürnberg), and all patients and controls included in this study signed an approved consent form. The animal experiments were approved by the regional government (Regierung von Unterfranken, Würzburg, Germany).

\section{Author contributions}

CD and JHWD designed the study. CD, YZ, SP, CB, XZ, ML, EK, and $\mathrm{AR}$ were involved in acquisition of data. $\mathrm{CD}, \mathrm{YZ}, \mathrm{SP}, \mathrm{CB}, \mathrm{ML}$, $\mathrm{TW}, \mathrm{EK}, \mathrm{AR}, \mathrm{OD}, \mathrm{GS}$, and JHWD were involved in interpretation of data. CD, TW, AR, OD, GS, and JHWD prepared the manuscript. KG, AY, and RJ provided essential material.

\section{Acknowledgments}

We would like to thank Christiane Bliß, Katja Dreißigacker, Regina Kleinlein, and Rita Weinkam for excellent technical support. The study was funded by grants DI 1537/7-1, DI 1537/8-1, DI 1537/9-1 and -2, DI 1537/11-1, DI 1537/12-1, DI 1537/13-1, DI 1537/14-1, DE 2414/2-1, DE 2414/4-1, RA 2506/3-1, RA2506/41, ZH 809/1-1, and AK 144/2-1 of the German Research Foundation; SFB CRC1181 (project C01) and SFB TR221/project number 324392634 (B04) of the German Research Foundation; grants J39, J40, and A64 of the IZKF in Erlangen; grants 2013.056.1 and 2017.129.1 of the Wilhelm-Sander-Foundation; grants 2014_A47, 2014_A248, and 2014_A184 of the Else-Kröner-Fresenius-Foundation; JSPS KAKENHI (S) JP17H06175 and AMED-CREST JP19gm1110009 (to AY); grant 14-12-17-1-Bergmann of the ELAN-Foundation Erlangen; and a Career Support Award of Medicine of the Ernst Jung Foundation.

Address correspondence to: Jörg H.W. Distler, Department of Internal Medicine 3, Universitätsklinikum Erlangen, Ulmenweg 18, 91054 Erlangen, Germany. Phone: 49.9131.35367; Email: joerg.distler@uk-erlangen.de. 
1. Gurtner GC, Werner S, Barrandon Y, Longaker MT. Wound repair and regeneration. Nature. 2008;453(7193):314-321.

2. Wynn TA. Cellular and molecular mechanisms of fibrosis. J Pathol. 2008;214(2):199-210.

3. Gabrielli A, Avvedimento EV, Krieg T. Scleroderma. N Engl JMed. 2009;360(19):1989-2003.

4. Varga J, Abraham D. Systemic sclerosis: a prototypic multisystem fibrotic disorder. J Clin Invest. 2007;117(3):557-567.

5. Massague J. TGFbeta signalling in context. Nat Rev Mol Cell Biol. 2012;13(10):616-630.

6. Strieter RM, Mehrad B. New mechanisms of pulmonary fibrosis. Chest. 2009;136(5):1364-1370.

7. Verrecchia F, Mauviel A. Transforming growth factor-beta and fibrosis. World J Gastroenterol. 2007;13(22):3056-3062.

8. Feghali CA, Wright TM. Identification of multiple, differentially expressed messenger RNAs in dermal fibroblasts from patients with systemic sclerosis. Arthritis Rheum. 1999;42(7):1451-1457.

9. Kikuchi K, et al. Growth regulation in scleroderma fibroblasts: increased response to transforming growth factor-beta 1.J Invest Dermatol. 1995;105(1):128-132.

10. Leask A, Abraham DJ. TGF-beta signaling and the fibrotic response. FASEB J. 2004;18(7):816-827.

11. Mori Y, Chen SJ, Varga J. Expression and regulation of intracellular SMAD signaling in scleroderma skin fibroblasts. Arthritis Rheum. 2003;48(7):1964-1978.

12. Sonnylal S, et al. Postnatal induction of transforming growth factor beta signaling in fibroblasts of mice recapitulates clinical, histologic, and biochemical features of scleroderma. Arthritis Rheum. 2007;56(1):334-344.

13. Leroy EC. Connective tissue synthesis by scleroderma skin fibroblasts in cell culture. J Exp Med. 1972;135(6):1351-1362.

14. Györfi AH, Matei AE, Distler JHW. Targeting TGF- $\beta$ signaling for the treatment of fibrosis. Matrix Biol. 2018;68-69:8-27.

15. Altorok N, Tsou PS, Coit P, Khanna D, Sawalha AH. Genome-wide DNA methylation analysis in dermal fibroblasts from patients with diffuse and limited systemic sclerosis reveals common and subset-specific DNA methylation aberrancies. Ann Rheum Dis. 2015;74(8):1612-1620.

16. Evans IC, et al. Epigenetic regulation of cyclooxygenase- 2 by methylation of c8orf 4 in pulmonary fibrosis. Clin Sci. 2016;130(8):575-586.

17. Hardy T, Mann DA. Epigenetics in liver disease: from biology to therapeutics. Gut. 2016;65(11):1895-1905.

18. Honda N, et al. miR-150 down-regulation contributes to the constitutive type I collagen overexpression in scleroderma dermal fibroblasts via the induction of integrin $\beta 3$. Am J Pathol. 2013;182(1):206-216.

19. O'Reilly S. Epigenetics in fibrosis. Mol Aspects Med. 2017;54:89-102.

20. Wang Y, Kahaleh B. Epigenetic repression of bone morphogenetic protein receptor II expression in scleroderma. J Cell Mol Med. 2013;17(10):1291-1299.

21. Jones PA. Functions of DNA methylation: islands, start sites, gene bodies and beyond. Nat Rev Genet. 2012;13(7):484-492.
22. Hermann A, Goyal R, Jeltsch A. The Dnmt1 DNA-(cytosine-C5)-methyltransferase methylates DNA processively with high preference for hemimethylated target sites. J Biol Chem. 2004;279(46):48350-48359.

23. Okano M, Bell DW, Haber DA, Li E. DNA methyltransferases Dnmt3a and Dnmt3b are essential for de novo methylation and mammalian development. Cell. 1999;99(3):247-257.

24. Zeisberg EM, Zeisberg M. The role of promoter hypermethylation in fibroblast activation and fibrogenesis. J Pathol. 2013;229(2):264-273.

25. Altorok N, Almeshal N, Wang Y, Kahaleh B. Epigenetics, the holy grail in the pathogenesis of systemic sclerosis. Rheumatology (Oxford). 2015;54(10):1759-1770.

26. Bergmann C, Distler JH. Epigenetic factors as drivers of fibrosis in systemic sclerosis. Epigenomics. 2017;9(4):463-477.

27. Dowson C, O'Reilly S. DNA methylation in fibrosis. Eur JCell Biol. 2016;95(9):323-330.

28. Zhang Y, et al. Poly(ADP-ribose) polymerase-1 regulates fibroblast activation in systemic sclerosis. Ann Rheum Dis. 2018;77(5):744-751.

29. Noda S, et al. Simultaneous downregulation of KLF5 and Fli1 is a key feature underlying systemic sclerosis. Nat Commun. 2014;5:5797.

30. Wang Y, Fan PS, Kahaleh B. Association between enhanced type I collagen expression and epigenetic repression of the FLI1 gene in scleroderma fibroblasts. Arthritis Rheum. 2006;54(7):2271-2279.

31. Fielding $C A$, et al. Interleukin- 6 signaling drives fibrosis in unresolved inflammation. Immunity. 2014;40(1):40-50.

32. Villarino AV, Kanno Y, O'Shea JJ. Mechanisms and consequences of Jak-STAT signaling in the immune system. Nat Immunol. 2017;18(4):374-384.

33. Chakraborty D, et al. Activation of STAT3 integrates common profibrotic pathways to promote fibroblast activation and tissue fibrosis. Nat Commun. 2017;8(1):1130.

34. Dees C, et al. JAK-2 as a novel mediator of the profibrotic effects of transforming growth factor $\beta$ in systemic sclerosis. Arthritis Rheum. 2012;64(9):3006-3015.

35 . Luo K. Signaling cross talk between TGF- $\beta / \mathrm{Smad}$ and other signaling pathways. Cold Spring Harb Perspect Biol. 2017;9(1):a022137.

36. Babon JJ, et al. Suppression of cytokine signaling by SOCS3: characterization of the mode of inhibition and the basis of its specificity. Immunity. 2012;36(2):239-250.

37. Babon JJ, Nicola NA. The biology and mechanism of action of suppressor of cytokine signaling 3 . Growth Factors. 2012;30(4):207-219.

38. Sasaki A, et al. Cytokine-inducible $\mathrm{SH} 2$ protein-3 (CIS3/SOCS3) inhibits Janus tyrosine kinase by binding through the $\mathrm{N}$-terminal kinase inhibitory region as well as $\mathrm{SH} 2$ domain. Genes Cells. 1999;4(6):339-351.

39. Babon JJ, Lucet IS, Murphy JM, Nicola NA, Varghese LN. The molecular regulation of Janus kinase (JAK) activation. Biochem J. 2014;462(1):1-13.

40. Lai RH, et al. SOCS6, down-regulated in gastric cancer, inhibits cell proliferation and colony formation. Cancer Lett. 2010;288(1):75-85.

41. Melzner I, et al. Biallelic deletion within 16p13.13 including SOCS-1 in Karpas1106P mediastinal
B-cell lymphoma line is associated with delayed degradation of JAK2 protein. Int J Cancer. 2006;118(8):1941-1944.

42. Mottok A, et al. Inactivating SOCS1 mutations are caused by aberrant somatic hypermutation and restricted to a subset of B-cell lymphoma entities. Blood. 2009;114(20):4503-4506.

43. Weniger MA, et al. Mutations of the tumor suppressor gene SOCS-1 in classical Hodgkin lymphoma are frequent and associated with nuclear phospho-STAT5 accumulation. Oncogene. 2006;25(18):2679-2684.

44. Inagaki-Ohara K, Kondo T, Ito M, Yoshimura A. SOCS, inflammation, and cancer. JAKSTAT. 2013;2(3):e24053.

45. Chen CY, et al. SOCS1 methylation in patients with newly diagnosed acute myeloid leukemia. Genes Chromosomes Cancer. 2003;37(3):300-305.

46. Chim CS, Fung TK, Cheung WC, Liang R, Kwong YL. SOCS1 and SHP1 hypermethylation in multiple myeloma: implications for epigenetic activation of the Jak/STAT pathway. Blood. 2004;103(12):4630-4635.

47. Chim CS, Wong KY, Loong F, Srivastava G. SOCS1 and SHP1 hypermethylation in mantle cell lymphoma and follicular lymphoma: implications for epigenetic activation of the Jak/STAT pathway. Leukemia. 2004;18(2):356-358.

48. Fourouclas N, et al. Methylation of the suppressor of cytokine signaling 3 gene (SOCS3) in myeloproliferative disorders. Haematologica. 2008;93(11):1635-1644.

49. Galm O, Yoshikawa H, Esteller M, Osieka R, Herman JG. SOCS-1, a negative regulator of cytokine signaling, is frequently silenced by methylation in multiple myeloma. Blood. 2003;101(7):2784-2788.

50. Huang L, et al. Transcriptional repression of SOCS3 mediated by IL-6/STAT3 signaling via DNMT1 promotes pancreatic cancer growth and metastasis. J Exp Clin Cancer Res. 2016;35:27.

51. Isomoto H, et al. Sustained IL-6/STAT-3 signaling in cholangiocarcinoma cells due to SOCS-3 epigenetic silencing. Gastroenterology. 2007;132(1):384-396.

52. Kim MH, et al. Suppressor of cytokine signaling (SOCS) genes are silenced by DNA hypermethylation and histone deacetylation and regulate response to radiotherapy in cervical cancer cells. PLOS ONE. 2015;10(4):e0123133.

53. Komazaki T, et al. Hypermethylation-associated inactivation of the SOCS-1 gene, a JAK/STAT inhibitor, in human pancreatic cancers. Jpn J Clin Oncol. 2004;34(4):191-194.

54. Niwa Y, et al. Methylation silencing of SOCS3 promotes cell growth and migration by enhancing JAK/STAT and FAK signalings in human hepatocellular carcinoma. Oncogene. 2005;24(42):6406-6417.

55. Pierconti F, et al. Epigenetic silencing of SOCS3 identifies a subset of prostate cancer with an aggressive behavior. Prostate. 2011;71(3):318-325.

56. Sutherland KD, et al. Differential hypermethylation of SOCS genes in ovarian and breast carcinomas. Oncogene. 2004;23(46):7726-7733.

57. Tischoff I, et al. Methylation of SOCS-3 and SOCS-1 in the carcinogenesis of Barrett's adenocarcinoma. Gut. 2007;56(8):1047-1053. 
58. Yoshikawa H, et al. SOCS-1, a negative regulator of the JAK/STAT pathway, is silenced by methylation in human hepatocellular carcinoma and shows growth-suppression activity. Nat Genet. 2001;28(1):29-35.

59 . He B, et al. SOCS- 3 is frequently silenced by hypermethylation and suppresses cell growth in human lung cancer. Proc Natl Acad Sci U S A. 2003;100(24):14133-14138.

60. Ogata $\mathrm{H}$, et al. Loss of SOCS3 in the liver promotes fibrosis by enhancing STAT3-mediated TGF-beta1 production. Oncogene. 2006;25(17):2520-2530.

61. Uhl M, et al. SD-208, a novel transforming growth factor beta receptor I kinase inhibitor, inhibits growth and invasiveness and enhances immunogenicity of murine and human glioma cells in vitro and in vivo. Cancer Res. 2004;64(21):7954-7961.

62. Yokogami K, Yamashita S, Takeshima H. Hypoxia-induced decreases in SOCS3 increase STAT3 activation and upregulate VEGF gene expression. Brain Tumor Pathol. 2013;30(3):135-143.

63. Bechtel W, et al. Methylation determines fibroblast activation and fibrogenesis in the kidney. Nat Med. 2010;16(5):544-550.

64. Asano Y. Epigenetic suppression of Fli1, a potential predisposing factor in the pathogenesis of systemic sclerosis. Int J Biochem Cell Biol. 2015;67:86-91.

65. Tabolacci E, et al. Genome-wide methylation analysis demonstrates that 5-aza-2-deoxycytidine treatment does not cause random DNA demethylation in fragile $\mathrm{X}$ syndrome cells. Epigenetics Chromatin. 2016;9:12.

66. Wang YY, et al. DNA hypermethylation of the forkhead box protein 3 (FOXP3) promoter in CD4+ T cells of patients with systemic sclerosis. Br JDermatol. 2014;171(1):39-47.

67. Rhead B, et al. Rheumatoid arthritis naive $\mathrm{T}$ cells share hypermethylation sites with synoviocytes. Arthritis Rheumatol. 2017;69(3):550-559.

68. van den Hoogen F, et al. 2013 Classification criteria for systemic sclerosis: an American College of Rheumatology/European League against Rheumatism collaborative initiative. Arthritis Rheum. 2013;65(11):2737-2747.

69. Beyer $C$, et al. $\beta$-catenin is a central mediator of pro-fibrotic Wnt signaling in systemic sclerosis. Ann Rheum Dis. 2012;71(5):761-767.

70. Dees C, et al. Platelet-derived serotonin links vascular disease and tissue fibrosis. J Exp Med. 2011;208(5):961-972.

71. Palumbo K, et al. The transcription factor JunD mediates transforming growth factor \{beta\}-induced fibroblast activation and fibrosis in systemic sclerosis. Ann Rheum Dis. 2011;70(7):1320-1326.

72. Akhmetshina A, et al. Activation of canonical Wnt signalling is required for TGF- $\beta$-mediated fibrosis. Nat Commun. 2012;3:735.

73. Palumbo-Zerr K, et al. Orphan nuclear receptor NR4A1 regulates transforming growth factor- $\beta$ signaling and fibrosis. Nat Med. 2015;21(2):150-158.

74. Wohlfahrt T, et al. PU.1 controls fibroblast polarization and tissue fibrosis. Nature. 2019;566(7744):344-349.

75. Distler JH, et al. Imatinib mesylate reduces production of extracellular matrix and prevents development of experimental dermal fibrosis. Arthritis Rheum. 2007;56(1):311-322.

76. Zerr P, et al. Vitamin D receptor regulates TGF- $\beta$ signalling in systemic sclerosis. Ann Rheum Dis. 2015;74(3):e20

77. Soare A, Ramming A, Avouac J, Distler JH. Updates on animal models of systemic sclerosis. J Scleroderma Relat Disord. 2016;1(3):266-276.

78. Yamamoto T, et al. Animal model of sclerotic skin. I: Local injections of bleomycin induce sclerotic skin mimicking scleroderma. J Invest Dermatol. 1999;112(4):456-462.

79. Akhmetshina A, et al. Treatment with imatinib prevents fibrosis in different preclinical models of systemic sclerosis and induces regression of established fibrosis. Arthritis Rheum. 2009;60(1):219-224.

80. Beyer C, et al. Stimulation of the soluble guanylate cyclase (sGC) inhibits fibrosis by blocking non-canonical TGF $\beta$ signalling. Ann Rheum Dis. 2015;74(7):1408-1416.

81. Dees C, et al. Stimulators of soluble guanylate cyclase (sGC) inhibit experimental skin fibrosis of different aetiologies. Ann Rheum Dis. 2015;74(8):1621-1625.

82. Dees C, et al. Inhibition of Notch signaling prevents experimental fibrosis and induces regression of established fibrosis. Arthritis Rheum. 2011;63(5):1396-1404.

83. Yasukawa H, et al. IL-6 induces an anti-inflammatory response in the absence of SOCS3 in macrophages. Nat Immunol. 2003;4(6):551-556.

84. Nguyen S, Meletis K, Fu D, Jhaveri S, Jaenisch R. Ablation of de novo DNA methyltransferase Dnmt3a in the nervous system leads to neuromuscular defects and shortened lifespan. Dev Dyn. 2007;236(6):1663-1676.

85. Armaka M, Apostolaki M, Jacques P, Kontoyiannis DL, Elewaut D, Kollias G. Mesenchymal cell targeting by TNF as a common pathogenic principle in chronic inflammatory joint and intestinal diseases. J Exp Med. 2008;205(2):331-337.

86. Horn A, et al. Inhibition of hedgehog signalling prevents experimental fibrosis and induces regression of established fibrosis. Ann Rheum Dis. 2012;71(5):785-789.

87. Minakuchi Y, et al. Atelocollagen-mediated synthetic small interfering RNA delivery for effective gene silencing in vitro and in vivo. Nucleic Acids Res. 2004;32(13):e109. 